\title{
Towards a new Liquid Argon Imaging Chamber for the MODULAr project
}

\section{Angeli, ${ }^{a}$ B. Baibussinov, ${ }^{b}$ M. Baldo Ceolin, ${ }^{c b}$ G. Battistoni, ${ }^{d}$ P. Benetti, ${ }^{e f}$}

A. Borio, ${ }^{e}$ E. Calligarich, ${ }^{f}$ M. Cambiaghi, ${ }^{e f}$ F. Cavanna, ${ }^{g h}$ S. Centro, ${ }^{c b}$ K. Cieslik, ${ }^{b}$ A. G. Cocco, ${ }^{i}$ R. Dolfini, ${ }^{e f}$ A. Gigli Berzolari, ${ }^{e f}$ C. Farnese ${ }^{c b}$ A. Fava, ${ }^{c b}$ A. Ferrari, ${ }^{k}$ G. Fiorillo, ${ }^{i c}$ D. Gibin, ${ }^{c b}$ A. Guglielmi, ${ }^{b}$ G. Mannocchi, ${ }^{l m}$ A. Menegolli, ${ }^{e f}$ G. Meng, ${ }^{b}$ C. Montanari, ${ }^{f}$ S. Muraro, ${ }^{d}$ O. Palamara, ${ }^{h}$ L. Periale,${ }^{l}$ P. Picchi, ${ }^{m}$ F. Pietropaolo,${ }^{b}$ A. Rappoldi, ${ }^{f}$ G. L. Raselli, ${ }^{f}$ M. Rossella, ${ }^{f}$ C. Rubbia,${ }^{h k *}$ P. Sala,${ }^{d}$ G. Satta, ${ }^{m}$ F. Varanini, ${ }^{c b}$ S. Ventura ${ }^{b}$ and C. Vignoli, ${ }^{f}$

${ }^{a}$ Dipartimento di Ingegneria Meccanica e Civile, Università di Modena e Reggio Emilia via Vignolese 905, I-41100 Modena, Italy

${ }^{b}$ INFN, Sezione di Padova via Marzolo 8, I-35131 Padova, Italy

${ }^{c}$ Dipartimento di Fisica, Università di Padova via Marzolo 8, I-35131 Padova, Italy

${ }^{d}$ INFN, Sezione di Milano via Celoria 2, I-20123 Milano, Italy

${ }^{e}$ Dipartimento di Fisica, Università di Pavia via Bassi 6, I-27100 Pavia, Italy

${ }^{f}$ INFN, Sezione di Pavia via Bassi 6, I-27100 Pavia, Italy

${ }^{g}$ Dipartimento di Fisica, Università dell'Aquila Via Vetoio, Località Coppito, I-67010 L'Aquila, Italy

${ }^{h}$ Laboratori Nazionali del Gran Sasso dell'INFN I-67010 Assergi (AQ), Italy

${ }^{i}$ INFN, Sezione di Napoli via Cintia, I-80126 Napoli, Italy

${ }^{j}$ Dipartimento di Scienze Fisiche, Università Federico II via Cintia, I-80126 Napoli, Italy

${ }^{k}$ CERN, European Laboratory for Particle Physics CH-1211 Geneve 23, Switzerland

${ }^{l}$ IFSI-INAF, Sezione di Torino Corso Fiume 4, I-10133 Torino, Italy

${ }^{m}$ Laboratori Nazionali di Frascati (INFN) via Fermi 40, I-00044 Frascati (RM), Italy E-mail: Carlo.Rubbiadcern.ch 
ABSTRACT: The MODULAr project foresees the exploitation of a new liquid Argon imaging detector, of at least $20 \mathrm{kt}$ fiducial mass, to be operated in a shallow depth location under the Gran Sasso Mountain. It will be devoted to study neutrino oscillations with an optimized off-axis CNGS neutrino beam. Cosmic neutrinos as well as proton decay will also be addressed. The MODULAr detector will vastly inherit from the technology developed for ICARUS-T600. However, such an increase in the volume over the current ICARUS-T600 needs to be carefully considered. It is concluded that a single, huge volume is an inoperable and uneconomical solution for many reasons. A very large mass is best realized with a modular set of many identical, independent units, each of about $5 \mathrm{kt}$, "cloning" the basic technology of the ICARUS-T600. Several of such modular units will be assembled to reach at least $20 \mathrm{kt}$ as initial sensitive volume. The increase of the active volume of about one order of magnitude with respect to the ICARUS-T600 detector requires some specific $\mathrm{R} \& \mathrm{D}$ activity, which will be implemented in a $\sim 360$ ton prototype unit (SLICE) of reduced length.

KEYWORDS: Large detector systems for particle and astroparticle physics; Liquid detectors; Particle tracking detectors; Time projection chambers.

${ }^{*}$ Corresponding author. 


\section{Contents}

1. Introduction 11

2. Main features of the new LAr-TPC detector

2.1 A modular approach

2.2 Double phase LAr-TPC signal collection?

2.3 A simplified structure for the modular detectors

2.4 The new experimental area 8

2.5 Initial filling procedures for the chamber

2.6 LAr purification 9

2.7 Photo-multipliers for light collection 11

2.8 Electronic readout and trigger 11

3. R\&D for MODULAr

3.1 LAr purification: from T300 to SLICE

3.2 Considerations on air evacuation procedures for the SLICE cryostat 16

3.3 Implementing the DAQ of MODULAr and SLICE

3.3.1 The ICARUS-T600 DAQ

3.3.2 Critical issues 21

3.3.3 The amplifier 2

3.3.4 The AD conversion 25

4. Conclusions 25

\section{Introduction}

The liquid Argon Time Projection Chamber (LAr-TPC), as imaging detector, was first proposed by C. Rubbia in 1977 [1]. In highly purified liquid Argon (LAr) ionizing tracks can be transported practically undistorted by a uniform electric field over macroscopic distances. A large set of wires at the end of a drift path continuously senses and records the signals induced by the drifting electrons. The signal of electrons crossing subsequent wire planes with different orientation is detected through non-destructive read-out of ionization electrons by charge induction. This provides several "bubble chamber like" projective views of the same event, allowing millimetre-like space point reconstruction and precise calorimetric measurements. The technique is especially suited for the detection of different kinds of neutrino events and other rare underground phenomena, like for instance proton decay, since the detector operates continuously and it does not require pressurization but only a "swimming pool" of cryogenic liquid. 
The feasibility of the technology has been demonstrated by the ICARUS collaboration with an extensive R\&D programme during more than two decades [2]. This gradual development of the technology has been completed by the industrial realization of a module employing about 600 tons of LAr (ICARUS-T600). The detector, made of two identical semi-modules, one of which has been successfully operated in 2001, is presently in its final position underground in the Hall B of the INFN Gran Sasso Laboratory (LNGS). It will collect simultaneously physics data with the long baseline neutrino beam from CERN (CNGS [3]) and from cosmic rays. All technical aspects of the system, namely cryogenics, LAr purification, read-out chambers, detection of LAr scintillation light, electronics and DAQ have been tested and perform as expected. A complete description of the construction and test of the ICARUS-T600 detector on surface has been given in [ $₫$ ].

Already in 2002, immediately after the successful operation of the ICARUS-T600, the Collaboration started to investigate even larger detectors in order to extend the physics results to even much larger masses [5]. The design of these developments is primarily motivated by the necessity of new detectors to complete the study of neutrino oscillations with beam and cosmic ray neutrinos, supernova explosions and the search for proton decay, especially in the exotic channels predicted by the SUSY theories. Programmes already under development in Japan and the US are the wellknown SuperKamiokande water Cherenkov detector [6] and the NOvA scintillator detector [7], both of the order of 20-30 kt mass. They are both intended to operate with off axis neutrino beams and for an optimum neutrino energy window of 1-2 GeV to study $v_{\mu} \rightarrow v_{e}$ sub-leading neutrino oscillations.

A LAr detector with an appropriate sensitive mass of about $20 \mathrm{kt}$, to be operated in a shallow depth location under the Gran Sasso Mountain in Italy (project MODULAr), has been recently proposed [8]. It may have significant advantages over the previously described options; in particular the active mass for a given sensitivity to neutrino oscillations is as much as a factor 4 smaller with respect to the ones of ref. [6] and [井].

The scientific community is presently also considering conceptual designs for huge "ultimate" detectors of the order of one or more hundreds of kilotons, whose huge costs are comparable to the ones of the LHC experiments: some R\&D is presently being pursued towards such a long distance goal. In Europe the following have been proposed: a bi-phase gas/liquid Argon TPC detector of a mass of $100 \mathrm{kt}$ exploiting charge amplification in the gas phase (GLACIER) [9], a huge liquid scintillation detector of about $50 \mathrm{kt}$ (LENA) [10] and a water Cherenkov counter of 440-730 kt [11] (MEMPHYS). In the US and in Japan two similar projects, UNO [12] and HyperKamiokande [13] have been discussed. It is remarked that also the presently described MODULAr detector [8] may be progressively expanded in order to reach eventually a sensitive mass which is comparable to the ones of the other "ultimate" detectors.

The ICARUS-T600 detector is providing an essential experience in order to develop such a "next step" with LAr. The large number of important milestones, which have been achieved in the last several years, has provided a solid background for the LAr-TPC. The operation of the ICARUS-T600 opens the way to the development of a new line of modular elements, which may be extrapolated progressively to the largest conceivable LAr-TPC sensitive masses.

One intermediate step towards the realization of the MODULAr project [8] is the construction of a relatively smaller test unit called SLICE, of much shorter length but otherwise identical to MODULAr in cross-section, with an active mass of about $360 \mathrm{t}$. 
An important amount of new physics results could be produced with this prototype, if exposed to an intense low energy neutrino beam such as those of the CERN-PS or the FNAL Booster. In particular, it would allow investigating the existence of sterile neutrinos and measure neutrino cross-section in the $0-3 \mathrm{GeV}$ energy range.

The present paper is devoted to the description of the innovative techniques of the MODULAr detector and the R\&D phase necessary for its successful achievement.

\section{Main features of the new LAr-TPC detector}

\subsection{A modular approach}

The most naïve design would assume a single LAr container of a huge size. But already in the case of containers of few thousand tons the geometrical dimensions of most types of events under study (beam- $v$, cosmic ray- $v$, proton decays) are relatively confined, i.e. much smaller than the fiducial volume. Hence, increasing the container's size does not affect appreciably the event acceptance in fiducial volume and does not introduce any significant physics arguments in its favour. We believe instead that there are a number of serious arguments for which such a huge size approach cannot be easily realised and that the use of a modular structure of several separate (similar) vessels, each of the size of a few thousand tons, provides a more reliable solution. For instance, in case of an accidental leak of the ultra-pure LAr, all the liquid in the actual volume of the container is contaminated. Segmentation is therefore useful in overcoming events due to poisoning of the liquid. In case of a major damage of the detector, the liquid can be provisionally transferred to another container. An additional reserve vessel of the order of $100 \mathrm{kt}$ is, on the other hand, not realistic. Moreover, the safety requirements of an underground vessel are strongly dependent on its size.

One of the most relevant features of LAr-TPC is its ability to detect accurately ionization losses at the percent level. Over a very large volume, the inevitable in-homogeneities in electron lifetime due to even modest variations in purity of the LAr produce very large fluctuations in the actual value of the collected charge and hamper the possibility of charge determination along the tracks. Therefore, we have chosen to use a modular approach of sufficient size in order to reduce the effects due to the non-uniformity of the electron collection generated by the emergence of negative ions, which impose a reasonably short maximum drift distance of each gap.

As it will be discussed further on, it has been assumed that a reasonable sensitive volume should be of $8 \times 8 \mathrm{~m}^{2}$ cross section and a length of about $60 \mathrm{~m}$, corresponding to $3840 \mathrm{~m}^{3}$ of liquid or $5370 \mathrm{t}$ of LAr. Two sets of readout chambers, placed at sides, and a central high voltage cathode are arranged in a double gap configuration with a drift length of $4 \mathrm{~m}$. The read-out chambers are made of three wire-planes with a wire pitch of the order of $6 \mathrm{~mm}$, twice the value of the ICARUS-T600.

It is well known that ionizing particles in LAr produce also a large amount of scintillation light

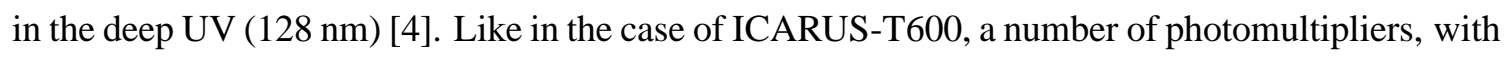
a thin layer of wave-length shifter (Tetra-Phenyl-Butadiene, TPB) deposited on the photocathode, will collect the light and extract a prompt signal for trigger and timing purposes. 


\subsection{Double phase LAr-TPC signal collection?}

Several years ago [14] the ICARUS collaboration had studied a double phase noble gas arrangement, in which ionization electrons from the tracks were drifted from the liquid to the superimposed gaseous phase. Electrons were further accelerated - with the production of proportional scintillation light - through a system of grids, like in an ordinary gaseous TPC, before being collected by the readout wire planes. Dark matter searches in Argon (WARP) [15] and in Xenon (XENON) [16] have profited from this technique.

Essentially the same idea has been also envisaged some time ago for very large ( $\geq 100 \mathrm{kt})$ monolithic LAr-TPC detectors. In the specific case of GLACIER [9], a very large electron drift length of $20 \mathrm{~m}$ at $1 \mathrm{kV} / \mathrm{cm}$ in LAr has been proposed. This corresponds to a drift voltage of as much as $2 \mathrm{MV}$, which is about a factor ten larger than the one discussed in the present proposal. If one assumes that the free electron lifetime is at least $2 \mathrm{~ms}$ [9], one expects an attenuation of the free electrons due to ion recombination in the impurities, presumably Oxygen, of as much as a factor 150 after $20 \mathrm{~m}$. This residual free electron component cannot be directly recorded electronically (as in ICARUS) over the whole drift distance and must be therefore first amplified by the proportional gain profiting of the gaseous phase. In practice, taking into account the large capacitance of the extremely extended read-out electrodes (up to $70 \mathrm{~m}$ long) and consequently of the larger noise signal from the input j-FET, gains of the order of $10^{3}$ are typical required values. Moreover, the dynamic range of the electronics must take into account the large variation of the signal pulse height along the drift coordinate.

Therefore, in the double phase arrangement, all three different types of charged particles have to be simultaneously considered, namely (1) the initial free electrons from LAr which are attenuated over the distance by as much as two orders of magnitude, (2) the accumulated negative ions from recombination by impurities and (3) the positive Argon ions especially from the multiplication near the wire in the gas. The ion speeds in liquid are extremely slow, typically far less than $2 \mathrm{~mm} / \mathrm{s},{ }^{1}$ at $1 \mathrm{kV} / \mathrm{cm}$ (with $2 \mathrm{MV}$ over $20 \mathrm{~m}$ the drift time is $\approx 10$ '000 s, i.e. about 3 hours!).

In the WARP and XENON experiments, it has been demonstrated that free electrons can overcome the liquid to gas barrier in the presence of a sufficiently strong electric field of a few $\mathrm{kV} / \mathrm{cm}$. It is expected that both the positive ions travelling from gas to liquid and the negative ions travelling from liquid to gas, because of their larger masses and hence smaller speed, will ultimately be trapped and accumulate at each side of the liquid-gas boundaries for a so far unknown period of time.

The free electrons crossing the double ion layer cloud are presently under study with the 2.3 litre WARP detector underground at the LNGS. There is some preliminary evidence, already in this small detector, that space charges, due to ions crossing the boundary, may introduce additional fluctuation in the electron ionization signal. This will introduce a substantial worsening of one of the most relevant features of LAr-TPC, namely its ability to detect accurately ionization losses.

The consequence for a detector of the size of 70 million litres and a diameter of $70 \mathrm{~m}$ is an enormous extrapolation, which still requires a considerable amount of R\&D. The phenomenon of ion transfer through the interface is expected to be rather complicated and not well understood and

\footnotetext{
${ }^{1}$ We remark that on such a timescale convective motions inside the vessel become very relevant and may seriously modify the drift time of positive ions in either direction.
} 
it has not been conclusively measured experimentally [17]. In the case relevant to GLACIER, it is likely that trapping may be ultimately occurring, but experimental studies are needed to assess the phenomenon. This is an absolutely crucial point prior to a successful, practical realization of a huge dual phase experiment in Argon.

As a first step, the $5 \mathrm{~m}$ long prototype ARGONTUBE [9] under study on surface should allow to experimentally verify these hypotheses and prove the feasibility of detectors with long drift paths. This represents a very important milestone in the conceptual proof of the feasibility of the dual phase detector in Argon.

It is therefore concluded that, at least at the present stage of the LAr-TPC, the single phase geometry and a relatively short drift length, which have both been already well developed experimentally [ [], are vastly preferable because they minimize the effects of negative and positive ions. Negative ions in this configuration are smoothly drifting and directly captured by the collecting wires with a negligibly small signal (the electric signal is proportional to the drift speed, a factor $\sim 10^{-5}$ smaller for ions). Positive ions are straightforwardly collected at the cathode. For a sufficiently small drift volume, like the one described in the present proposal, the electric field distortions due to the slow ion motion can be made negligible.

\subsection{A simplified structure for the modular detectors}

The structure of the detector has been considerably streamlined in order to reduce the number of components, its cost and to increase the reliability of the system. The modular structure permits to repeat the initial engineering design of the prototype in a series of several subsequent units, reducing progressively both cost and construction time.

One of the goals of the detector is related to filling and maintaining over many years a very large amount of ultra-pure LAr in stable conditions inside a dedicated underground cave, within very strict safety conditions. The initial filling procedure is determined by the supply rate currently provided by the supplying industry, the maximum rate available in the European market being of the order of $200 \mathrm{~m}^{3} / \mathrm{d}$. At the rate of $100 \mathrm{~m}^{3} / \mathrm{d}$, the initial filling of $21.5 \mathrm{kt}$ of ultra-pure LAr (corresponding to a volume of $15^{\prime} 500 \mathrm{~m}^{3}$ ) is about 155 days, which is acceptable. At the commercial cost of 0.7 Euro/l, the value of the LAr for the initial filling is about 11 MEuro, which is also quite acceptable. The mechanical structure of each of the modular units should be as simple as possible, keeping the cost of the various components commensurate with the relatively modest initial investment for the LAr.

The wire arrangement is scaled from the industrial realization of the existing ICARUS-T600, which is taken as reference design. ICARUS-T600 is made of two identical T300 modules, in which two sets of readout planes and a high voltage cathode are located in a double gap configuration. Each set of readout planes is made of three parallel wire planes (separated by a gap of $3 \mathrm{~mm}$ ), the wires on each plane are stretched at $0^{\circ}$ and $\pm 60^{\circ}$ with respect to the horizontal direction. The most inner wire plane (facing the drift volume) acts essentially as a screen grid for the successive induction plane. However the signals induced on the horizontal wires are also used to solve possible ambiguities in the three dimensional track reconstruction. This identical geometry is "cloned" into a larger modular detector, with the linear dimensions scaled by a factor $8 / 3=2.66$, namely the cross sectional area of the planes is now $8 \times 8 \mathrm{~m}^{2}$ rather than $3 \times 3 \mathrm{~m}^{2}$. The wires in the longitudinal direction were originally $9.4 \mathrm{~m}$ long with the wire planes subdivided into two equal segments. In the 


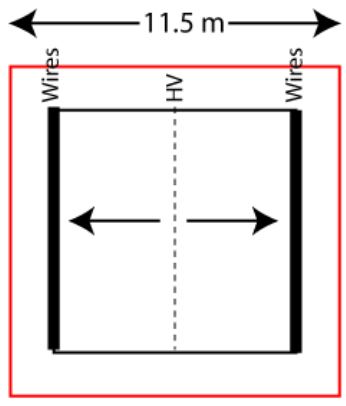

(a) Type T300 with $4 \mathrm{~m}$ drift 5'000 ton and 25'000 wires

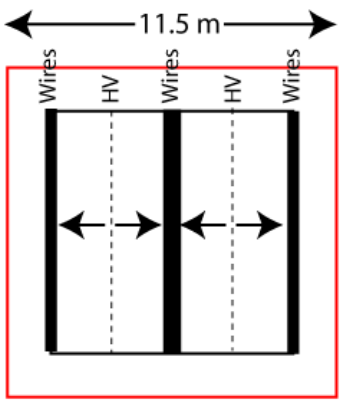

(b) Type T300 with $2 \mathrm{~m}$ drift 5'000 ton and 50'000 wires

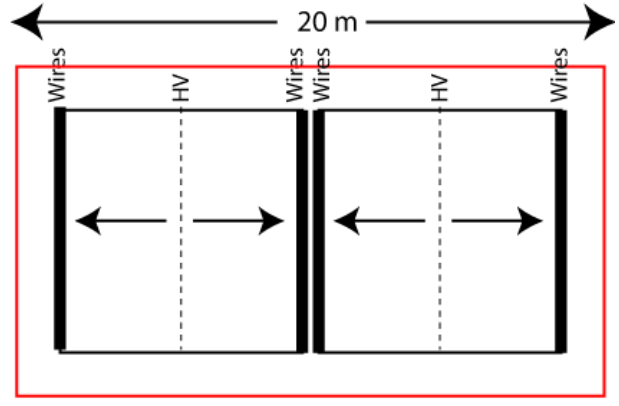

(c) Type T600 with $4 \mathrm{~m}$ drift $10^{\prime} 000$ ton and $50^{\prime} 000$ wires

Figure 1. Various possible alternative arrangements for a modular unit, with a transverse cross-section of $8 \times 8 \mathrm{~m}^{2}$ for each module. In (a) we show a scaled up T300 configuration with $4 \mathrm{~m}$ drift length and about $25^{\prime} 000$ readout wires. The sensitive mass is about 5000 ton. In (b) the configuration is the same as in (a), except the drift distance has been reduced to $2 \mathrm{~m}$ doubling the number of readout wires. In (c) we show a scaled up ICARUS-T600 configuration with a twin module, $4 \mathrm{~m}$ drift length and about 50'000 wires. The sensitive mass is then $10^{\prime} 000$ tons.

next step, the length will be also split into two individual wire sets, but $30 \mathrm{~m}$ long, corresponding again to the ratio $30 / 9.4=3.2$. The longer wires have a higher capacitance and the signal/noise ratio is significantly decreased (wires, of the order of $10 \mathrm{pF} / \mathrm{m}$; cables, of the order of $50 \mathrm{pF} / \mathrm{m}$ ). This factor is compensated widening the pitch to $6 \mathrm{~mm}$, to be compared to the $3 \mathrm{~mm}$ adopted in ICARUS-T600, thus doubling the $d E / d x$ signals. Therefore, we expect signal/noise ratios which are rather similar to the ones of the ICARUS-T600, namely of the order of 10/1. As it will be discussed later on, over the last several years the electronic chain from "wire to computer" has been considerably improved in performance and reduced in $\operatorname{cost}^{2}$, in collaboration with industry.

Each stereo wire is observing a time projected volume which is a factor $2.66 \times(4 / 1.5) \times 2=$ 14.2 larger than in the case of the ICARUS-T600 (wire length $\times$ drift length $\times$ wire pitch). The average LAr mass observed by each TPC readout wire is about $200 \mathrm{~kg} /$ channel. Therefore a $20 \mathrm{kt}$ sensitive volume will require of the order of $10^{5}$ wires.

At this stage the configuration of the modules may not be considered as final and a number of configurations are possible, as shown in figure 1, maintaining as a reference a transverse crosssection of $8 \times 8 \mathrm{~m}^{2}$.

Figure 17a represents the previously indicated basic configuration of a scaled T300 double gap arrangement. The nominal voltage of the $\mathrm{T} 300$ is $75 \mathrm{kV}$ for the $1.5 \mathrm{~m}$ long drift, corresponding to a drift field of $500 \mathrm{~V} / \mathrm{cm}$. However, the field-shaping electrodes have been designed and safely tested for cathode $\mathrm{HV}$ values of more than $200 \mathrm{kV}$, in view of a drift length extension up to $3 \mathrm{~m}$ as required in a never constructed T1200 module. The T300 has been currently operated without problems with $\mathrm{HV}$ up to $150 \mathrm{kV}$ (i.e. a drift field of $1 \mathrm{kV} / \mathrm{cm}$ ).

In MODULAr, the HV on the cathode would be $200 \mathrm{kV}$, at a nominal electric field of $500 \mathrm{~V} / \mathrm{cm}$

\footnotetext{
${ }^{2}$ The estimated commercial cost of each channel "wire to computer" is now about 60 Euro. Assuming for each wire a sensitive LAr volume of $200 \mathrm{~kg}$, the "cost" of the electronics is $60 / 200=0.3 \mathrm{Euro} / \mathrm{kg}$, which is about $1 / 2$ of the cost of the Argon procurement. The low cost achieved for such a sophisticated electronics is an additional argument in favour of a single phase LAr configuration with a relatively short drift gap distance, along the lines of the T600, rather than the double phase GLACIER arrangement.
} 

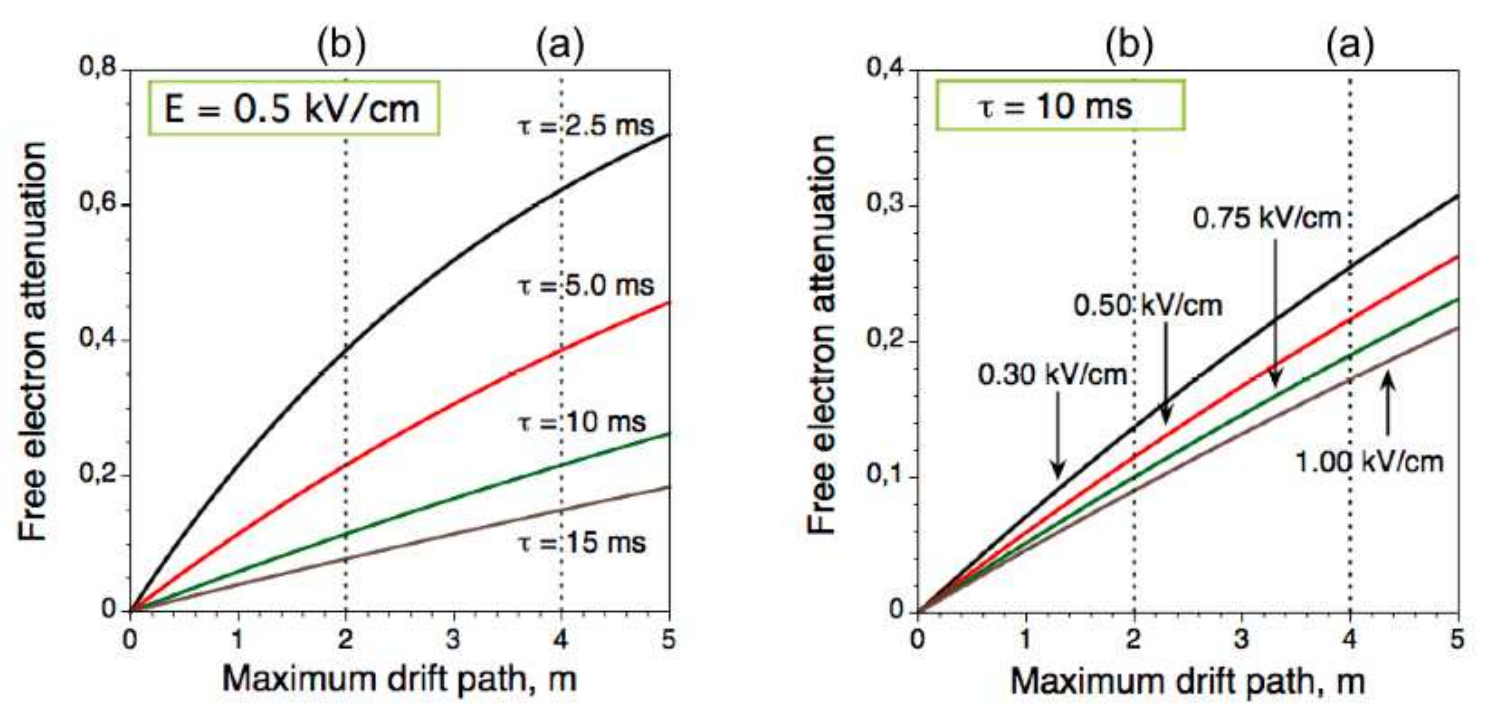

Figure 2. The free electron attenuation is shown as a function of the maximum electron drift path, for different values of the free electron lifetime, $\tau$, at $\mathrm{E}=0.5 \mathrm{kV} / \mathrm{cm}$ (left) and for different electric drift fields and a $10 \mathrm{~ms}$ electron lifetime (right). Electron drift velocities have been deduced from ref. [†]. For the electric fields assumed in the figure, the corresponding electron drift velocities are: $1.16 \mathrm{~mm} / \mu \mathrm{s}(0.3 \mathrm{kV} / \mathrm{cm})$, $1.56 \mathrm{~mm} / \mu \mathrm{s}(0.5 \mathrm{kV} / \mathrm{m}), 1.84 \mathrm{~mm} / \mu \mathrm{s}(0.75 \mathrm{kV} / \mathrm{m}), 2.05 \mathrm{~mm} / \mu \mathrm{s}(1.0 \mathrm{kV} / \mathrm{m})$. Cases (a) and (b) represent respectively the configurations of figures $1 \mathrm{a}$ and $1 \mathrm{~b}$ with $4 \mathrm{~m}$ and $2 \mathrm{~m}$ drift paths.

and $4 \mathrm{~m}$ drift. However, the electron drift time, $\mathrm{t}_{\text {drift }}$, will be shortened with a significantly higher field, since $t_{\text {drift }} \propto\left(\mathrm{E}_{\text {drift }}\right)^{-0.5}$. The requirements of purity for the LAr will be correspondingly reduced. For instance a $\mathrm{HV}$ of $350 \mathrm{kV}$ will provide a maximum electron drift time equal to the one already optimized of a T1200 module with a $3 \mathrm{~m}$ drift.

In figure $1 \mathrm{~b}$ the number of wire planes has been doubled in order to reduce the drift distance to $2 \mathrm{~m}$. However, in this configuration, the number of signal wires is also doubled to $50^{\prime} 000$, with a significant increase in the cost of the electronic channels. In order to maintain the maximum electron drift time equal to the one of the T300, an increase of the drift field to $75 \mathrm{kV} \times$ $(2 / 1.5)^{3}=178 \mathrm{kV}$ is needed, a value which is at reach even with the present T300 field shaping design. Although we believe that the drift distance can be safely extended to $4 \mathrm{~m}$, this alternative shows that the choice of the electron drift length is not critical and could be adopted as needed.

Finally, figure 1]c shows a scaled up ICARUS-T600 twin volume configuration, with $4 \mathrm{~m}$ drift time and about 50'000 wires. The two volumes are physically separated, but they are both kept in the same cryogenic volume. The total sensitive mass of one module of type (c) in figure 1 is then 10 '000 t. The new halls of the LNGS, proposed for the MODULAr project [8], could host two modules of type $1 \mathrm{c}$ for a total of $20 \mathrm{kt}$.

Considerable experience in the ICARUS collaboration has shown that free electron drift times of several milliseconds are currently realised with commercial purification systems based on Oxysorb ${ }^{\mathrm{TM}}$. The effects on the electron attenuation are shown in figure 2 where the charge attenuation versus drift path at different electric fields are given for $\mathrm{t}_{\text {drift }}=10 \mathrm{~ms}$ and for different electron lifetimes at $0.5 \mathrm{kV} / \mathrm{cm}$. 
The r.m.s. spread $\sigma_{D}$ of the free electron cloud is $\sigma_{D}=\left(2 \mathrm{D} x / v_{D}\right)^{0.5}$, both for longitudinal and transverse diffusion, where $\mathrm{D}=4.06 \mathrm{~cm}^{2} \mathrm{~s}^{-1}, x$ is the drift path and $\mathrm{v}_{D}$ is the drift velocity. In more practical units, $\sigma_{D}[\mathrm{~mm}] \approx 0.9\left(t_{\text {drift }}[\mathrm{ms}]\right)^{0.5}$. For a drift field of $0.5 \mathrm{kV} / \mathrm{cm}$ and a $4 \mathrm{~m}$ path the average value is $\left\langle\sigma_{D}\right\rangle \approx 0.9 \mathrm{~mm}$ and the maximum value is $\sigma_{\text {Dmax }} \approx 1.4 \mathrm{~mm}$. As a consequence, the spatial resolution in the MODULAr detector, in which a wire pitch of $6 \mathrm{~mm}$ is foreseen, is weakly affected by diffusion even in the case of maximum drift distance.

The new mechanical structure, which has been highly streamlined, is essentially made of only three main mechanical components:

- An external insulating vessel made of two metallic concentric volumes, filled in between with perlite. Perlite is a mineral, which is vastly used industrially, the world annual consumption being of the order of 2 million tons. ${ }^{3}$ The environmental aspects of perlite are not severe: mining generally takes place in remote areas, and airborne dust is captured by bag-houses, and there is practically no runoff that contributes to water pollution. In order to ensure an adequate thermal insulation, about $1.5 \mathrm{~m}$ thickness is required, corresponding to over $3000 \mathrm{~m}^{3}$ for a container. The bottom-supporting layer could be made out of low conductivity light bricks. The specific heat loss of perlite is $3.86 \mathrm{~W} / \mathrm{m}^{2}$ for a nominal thermal conductivity of $0.029(0.025-0.029) \mathrm{W} / \mathrm{m} / \mathrm{K}$. This is significantly smaller than the specific heat loss of the ICARUS-T600. Taking into account the dimensions of the vessel, the total heat loss is 8.28 $\mathrm{kW}$. At present in the LNGS the cryogenic plant of ICARUS-T600 is made of 10 units, each with $4 \mathrm{~kW}$ of (cold) power. Three of these units $(\leq 12 \mathrm{~kW})$ should be adequate to ensure cooling of the walls of the vessel during normal operation. Evacuation of the perlite is therefore unnecessary.

- A self-supporting structure holding the frame with the wire planes at each lateral side and the high voltage plane at the centre. The photomultipliers for the scintillation light detection and trigger purposes are also mounted on this frame, behind the wire planes. The structure of the planes is identical to the one already developed for the ICARUS-T600, except that only one wire out of two is installed in order to go from a $3 \mathrm{~mm}$ to $6 \mathrm{~mm}$ pitch. The inner structure of the huge container is therefore extremely simple, being primarily a linear wire structure along the edges of the container, the rest remaining essentially free of structures.

- The LAr and N2 supply and refrigeration, provided with cooling and purification both in the liquid and gas phases, with an appropriate re-circulating system to ensure that the whole liquid is moving orderly inside the vessel volume to ensure the uniformity of the free electron lifetime.

\subsection{The new experimental area}

The ICARUS-T600 detector is located inside the Hall B of the LNGS laboratory in an appropriate containment tank constructed above the floor of the Hall. The new experimental area, indicated as LNGS-B, could be realized about $10 \mathrm{~km}$ off-axis from the main laboratory, away from the protected area of the Gran Sasso National Park. A provisional location is under consideration, protected by

\footnotetext{
${ }^{3}$ The present production in Greece, where vast resources are available, is about $500 \mathrm{kt} / \mathrm{y}$. The estimated cost is about $40 \$ / \mathrm{t}$
} 
$1.2 \mathrm{~km}$ of equivalent water depth. The excellent imaging capability of the LAr-TPC detector will ensure the absence of backgrounds not only for the neutrinos from the CNGS but also for proton decay and cosmic neutrinos.

It is foreseen that the size of the new LNGS-B facility could be about $50^{\prime} 000 \mathrm{~m}^{3}$, slightly larger than the present Hall-B. However, differently from the main LNGS, the shapes of the cavities, rather than being vast, general-purpose halls, would be tailored to the specific experiment.

As already described [8], each MODULAr unit is located in an appropriate "swimming pool" cave surrounded by perlite walls, thus avoiding any realistic possibility of leaks outside the walls, The worst case is a total loss of the external cryogenic cooling liquids (Nitrogen/Argon). The tank will spontaneously warm up in contact with the heat leaks of the surrounding components through the $1.5 \mathrm{~m}$ thick perlite wall. At a heat leak rate of $10 \mathrm{~kW}$, the LAr evaporation rate is of $220 \mathrm{~kg} / \mathrm{h}$, which is negligibly small with respect to the $5 \mathrm{kt}$ of the stored LAr tank. The tank will remain stable in its liquid form over a long time. Moreover, there is no possibility that the environment around the cave would provide a sufficiently large amount of heat causing a catastrophic evaporation of a massive amount of LAr.

For a configuration of the type $1 \mathrm{c}$, the cross section is $11 \times 20 \mathrm{~m}^{2}$ (shown in figure 3 ) and the length is about $60 \mathrm{~m}$. Different modules may have entirely separate halls since the event containment is anyway very good. An exhaust pipe is necessary to evacuate the evaporated liquid into the atmosphere in case of an accidental leak, although the probability of such events is very small, as indicated in the risk analysis performed for ICARUS considering a $3 \mathrm{kt}$ detector mass [5].

\subsection{Initial filling procedures for the chamber}

In the present ICARUS-T600 the vessel is evacuated before injecting ultra pure Argon. Since the new detector is very hard to evacuate, in view of its large size, a new method has to be adopted. The idea is to perform successive flushing in the gaseous phase in order to attenuate the presence of gases other than Argon in an approximately exponential manner. This method of flushing with pure Argon gas (GAr) is already widely used in gaseous wire and drift chambers, which are generally not evacuated. In the present case, additional problems may arise in view of the magnitude of the volume and the possibility of creating, for instance, "dead" spots where the gas may not circulate.

An average surviving gaseous volumetric concentration of Oxygen equivalent impurities of the order of $10^{-6}(1 \mathrm{ppm})$ is a reasonable goal. As a next step, the volume will be cooled down and filled with ultra-pure LAr, with the surviving relative concentration of LAr reduced by the obvious factor $1 / 835$ - the density of the GAr at room temperature being about 835 times smaller than the one of the liquid - leading to an Oxygen equivalent impurity concentration in the liquid phase of about $1.2 \mathrm{ppb}$. Such level of impurities corresponds to an initial LAr electron lifetime of about a few hundred microseconds (1 ppb of Oxygen equivalent gives an electron lifetime of $300 \mu \mathrm{s}$ ), which will be further improved with the help of the OXISORB/HYDROSORB system. On the basis of previous experience, a final electron lifetime of about $10 \mathrm{~ms}$ should be reachable. This value corresponds to a contamination equivalent to $\sim 30 \mathrm{ppt}$ of Oxygen and an electron signal attenuation of about $20 \%$ for the longest, $4 \mathrm{~m}$ drift distance.

\subsection{LAr purification}

A vigorous, continuous purification, through re-circulation of the LAr, must be performed with 


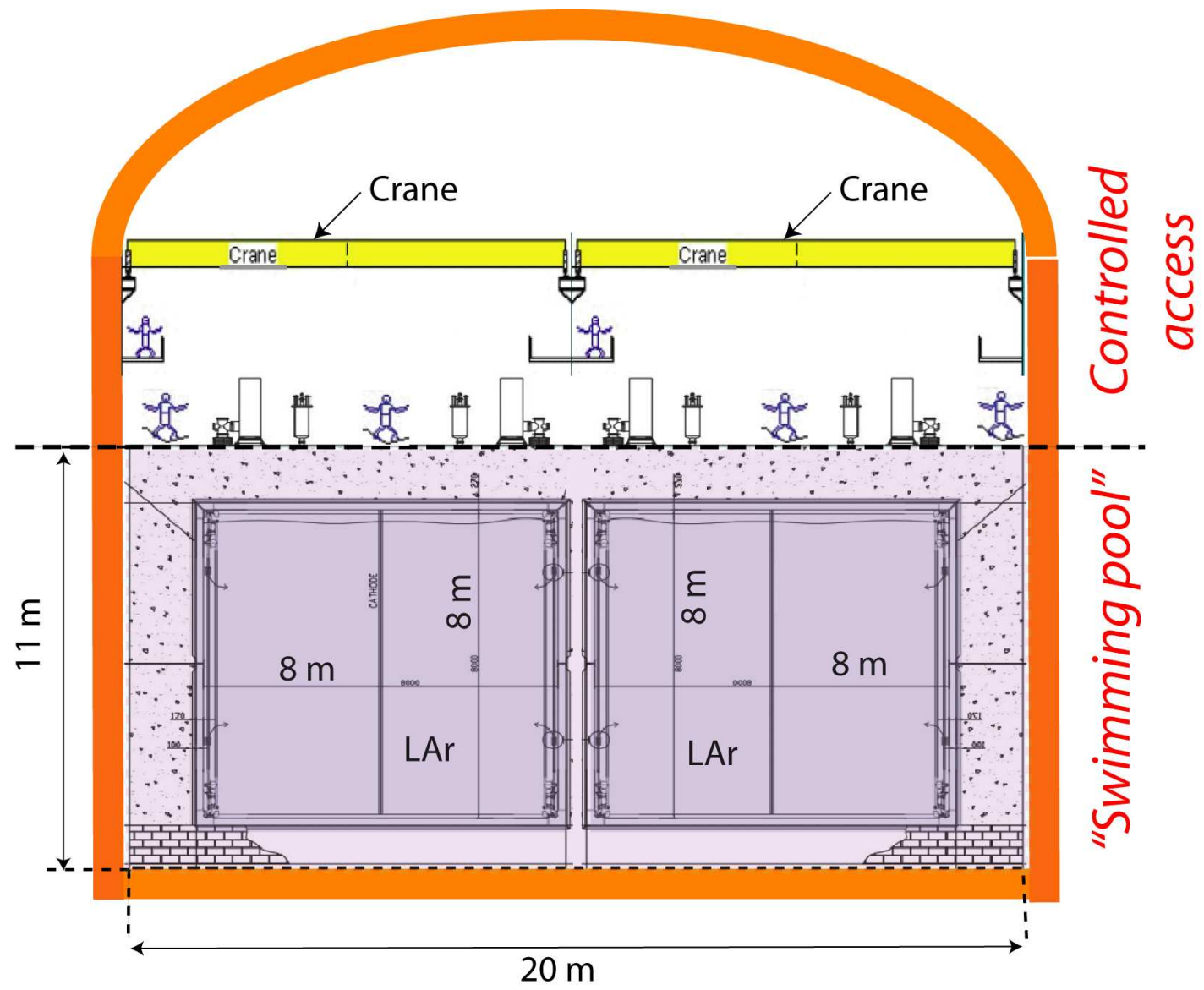

Figure 3. Indicative cross section of the ICARUS-T600 "clone" in the dedicated "swimming pool like" underground hall. The lower part is made of two twin separate LAr containers made of Aluminium extruded structures, thermally stabilized with forced N2 circulation. Outside the structure a $1.5 \mathrm{~m}$ thick perlite wall provides spontaneous, passive heat insulation. The region on top of the "swimming pool" is accessible to auxiliary equipments. Personnel access is strictly controlled.

filtering methods based on OXISORB ${ }^{\mathrm{TM}}$ and molecular sieves, in order to ensure a free electron lifetime adequate for the longest $\approx 3 \mathrm{~ms}$ drift time. In analogy with ICARUS-T600 and all previously constructed detectors, the purification is performed re-circulating both the liquid and the gaseous phases. The liquid re-circulation rate needed to reach safely the running condition in few months is of the order of several percent of volume per day. For a volume of $4000 \mathrm{~m}^{3}$, this means a re-circulation rate of the order of $240 \mathrm{~m}^{3} /$ day (i.e.: $6 \%$ per day or one full detector volume re-circulated in about 17 days), in the range which is possible with the ICARUS-T600 purification scheme. ${ }^{4}$

In conclusion, a volume of a few thousand cubic metres is a reasonable limit for a single LArTPC. It could be cleaned, cooled and filled in few months and the required LAr purity could be reached in few more months. Altogether, a detector of this size could be put in operation in a reasonable period of time.

\footnotetext{
${ }^{4}$ The standard rate of a single OXISORB ${ }^{\mathrm{TM}}$ pack is about $120 \mathrm{~m}^{3} / \mathrm{d}$. Therefore two of such units are sufficient for the chosen size of the vessel.
} 


\subsection{Photo-multipliers for light collection}

Like in the case of ICARUS-T600 a number of photomultipliers located behind the readout wires are used to provide a trigger signal and timing. These photomultipliers are necessary for the cosmic rays and proton decay events to provide a starting signal. They could as well be very useful in order to tag events coming from the CNGS beam. The technique already used in the case of the ICARUST600 and in the WARP experiment, consists in using large area glass phototubes working at LAr temperature [4] equipped with a thin deposit of wave-length shifter (Tetra-Phenyl-Butadiene, TPB). The scintillation light from the LAr, emitted in the deep UV (128 nm) is converted to the visible range with an efficiency close to $100 \%$. A significant contribution to the recorded light is also given by the Cherenkov light emitted in the visible wavelength range by relativistic particles.

\subsection{Electronic readout and trigger}

The DAQ system $\left(5 \cdot 10^{4}\right.$ channels) of the ICARUS-T600 detector is based on an analogue front-end followed by a multiplexed 10-bit ADC and by a digital VME module that performs local storage and data compression. It has been tested satisfactorily in the 2001 run [4. In a multi-kton LAr-TPC the $6 \mathrm{~mm}$ pitch foreseen for the wires (or in general electrodes) will allow using most of the tools already built and designed for the ICARUS-T600.

The present ICARUS Integrated Circuit is used for the front-end amplifier. A silicon run of the specific BiCMOS process has been recently made, allowing to equip up to $1.410^{5}$ electronic channels. A R\&D program is also proposed for the development of a hybrid sub module hosting eight or more channels of amplification and eventually, as it will be described later, also the analogue to digital converter. At present a revision of the ICARUS analogue electronics is underway with the aim of further improving the front-end performance.

A novel technique for the realization of feed-through's has been developed. INFN holds a patent (RM2006A000406) for this technology that allows easy realization of feed-through's with high-density vias and different shapes.

As described in more detail in section 3.3, four 10-bit ADC's, grouped in two sets, work at $20 \mathrm{MHz}$ sampling rate on multiplexed (1:8) amplifier outputs, namely each channel is sampled every 400 ns. Two ADC's outputs are combined in a 20-bit word. The two sets of two ADC's are transmitted interleaved; hence the 20-bit digital output has a $40 \mathrm{MHz}$ frequency. The power dissipated is significant: $500 \mathrm{~mW}$. The required bandwidth is $800 \mathrm{Mb} / \mathrm{s}$ for 32 channels.

The main feature of the new design is to move into the digital domain all the conversion processes at a very early stage and to exploit the use of numerical digital filtering techniques. The trigger system will divide the detector in sub-volumes to deal with the data acquisition rate imposed by the shallow depth location. The basic structure of the trigger system will reproduce that of the ICARUS-T600.

\section{R\&D for MODULAr}

The increase of the active LAr volume of about one order of magnitude with respect to ICARUST600, the streamlining and simplification of the mechanical structures and the new developments previously described in the structure of the detector require some specific R\&D developments. 


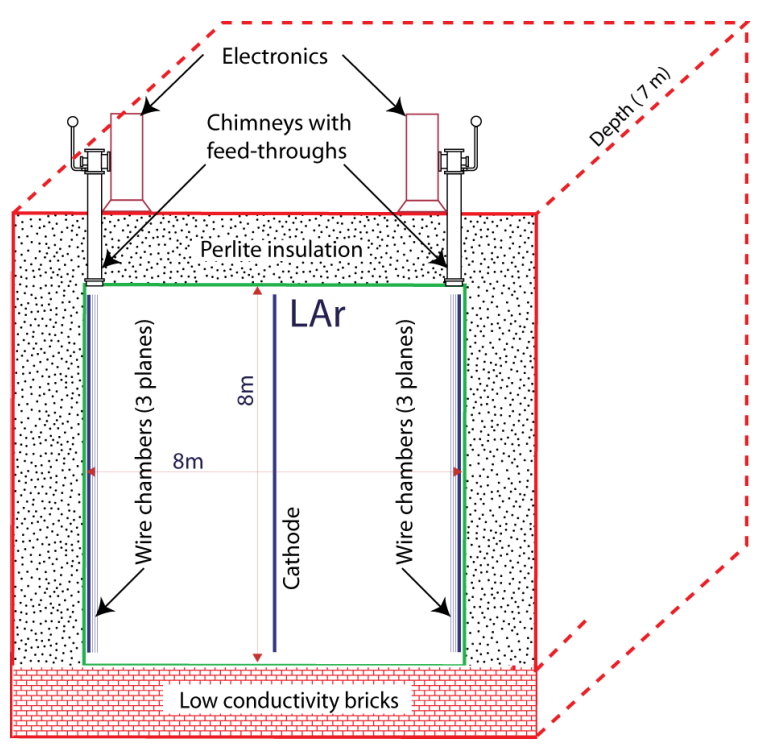

Figure 4. Schematics of the SLICE detector.

However these are not of very substantial nature and could be implemented in parallel with the detailed engineering design of MODULAr. These developments are intended to simulate on a small scale and investigate the basic innovations introduced with respect to the ICARUS-T600 solutions, namely:

- the filling process starting from air to pure LAr, taking into account the motion of the gas, optimizing the inlet and outlet geometries and minimizing the number of cycles;

- the thermal convection of the LAr, in order to optimize the temperature gradients and to ensure a convincing circulation in all regions of the dewar, both in the cool down and stationary phases;

- the out-gassing rate and the re-circulation processes necessary to achieve the required electron lifetime;

- the geometry of the compact re-circulators both in the liquid and in the gaseous phases.

Finally, also the electronics and DAQ may require some specific developments to improve the layout of the analogue front-end and the DAQ architecture.

A prototype unit of reduced longitudinal dimension, but otherwise identical to the final MODULAr unit, must be constructed and tested. Called "SLICE" (see figure $\bigoplus$ ), it is transversally $(x, y)$, identical to the final MODULAr unit (configuration (a) of figure 11). It has a sensitive area of $8 \times 8 \mathrm{~m}^{2}$ with a pair of three readout planes $\left(0^{\circ},+60^{\circ},-60^{\circ}\right)$ one at each side of the gap and a central HV plane and a maximum drift distance of $4 \mathrm{~m}$. However, it is much shorter than MODULAr, in the $(z)$ direction, namely about $4 \mathrm{~m}$ of active width. The electric field is made uniform with the help of field shaping rings similar to those used in the ICARUS-T600 LAr-TPC.

The resulting sensitive volume of SLICE is of the order of $264 \mathrm{~m}^{3}$, corresponding to $360 \mathrm{t}$ of LAr, roughly the mass of the T300 module [ $\llbracket$. The main components of SLICE are the following: 
- The perlite insulation is contained in an outer protecting box, presumably concrete blocks, simulating the underground swimming pool structure of the underground detector. The volume of expanded perlite is about $550 \mathrm{~m}^{3}$, corresponding to a mass of $33 \mathrm{t}$ at an expanded perlite density of 60 (48 to 72$) \mathrm{kg} / \mathrm{m}^{3}$.

- The inside walls of the perlite container are a thick extruded Aluminium box, surrounding the whole inner detector structure, through which the cooling N2 is circulated, to ensure a high level of temperature uniformity to the inner body.

- In ordinary conditions, the LAr flow must proceed orderly and uniformly, for instance from bottom to top across the full $>8 \mathrm{~m}$ height of the liquid, in order to avoid possible "pockets" of limited circulation which could persist for a long time due to the very slow diffusion of the liquid. To this effect, a bottom containment plate with many small holes uniformly distributed over the whole surface ensures a uniform flow from bottom to top of the LAr volume. Likewise, in the boundaries between the liquid and gaseous phase, an appropriate "gutter" structure is conveying uniformly the liquid exiting the volume.

- A continuous purification must be performed both in the liquid and in the gaseous phases, with the help of molecular sieves and $\mathrm{O}_{2}$ absorbers, as described later on. The purifiers are a set of tubes placed outside the perlite vessel and connected with the help of appropriate pumps to the inner LAr volume as discussed in point 3.

- A reasonable anode structure with three-wire plane chambers will have a sampling pitch of the order of $6 \mathrm{~mm}$, twice the value adopted for ICARUS-T600. The wire planes are "cloned" from the ICARUS-T600 construction and T1200 detailed engineering designs with a wire pitch of $3 \mathrm{~mm}$ and a plane separation of $3 \mathrm{~mm}$ as well, in order to preserve the signal pulse shape. The length of the wires of SLICE is comparable to the one of ICARUS-T600 and therefore the mechanical construction is very straightforward. The $6 \mathrm{~mm}$ sampling pitch is simply obtained joining pairs of adjacent wires in this structure.

- The number of read-out channels is about 7700 , a relatively small fraction of the T300 readout system. The required memory depth, due to the $4 \mathrm{~m}$ drift, is also fitting the present T300 DAQ design, which could be used "as it is". Nevertheless SLICE could be the benchmark for the implementation of new read-out improvements as foreseen for MODULAr.

- The nominal voltage of the $\mathrm{T} 300$ is $75 \mathrm{kV}$ for the $1.5 \mathrm{~m}$ long drift, corresponding to a drift field of $500 \mathrm{~V} / \mathrm{cm}$, although the racetrack has been currently operated without problems up to $150 \mathrm{kV}$. At the same nominal electric field $(500 \mathrm{~V} / \mathrm{cm})$, for the present choice of SLICE, the HV would be $200 \mathrm{kV}$.

- Photomultipliers, coated with TPB wavelength shifter, located behind the readout wires are also foreseen to provide a trigger signal and timing.

- The initial filling and purification of the LAr is one of the main new features of the MODULAr technology. As described before, the method consists of an initial filling of the volume with ultra-pure gaseous Ar, following the well-known technique of repeated flushing of the gas. 


\subsection{LAr purification: from T300 to SLICE}

The purification system of the T300 module consists of two separated circuits for the liquid and the gaseous phases. The first is used for the initial filling with commercial LAr and for its recirculation during ordinary detector operation, to guarantee an adequate and stable LAr purity. The second circuit, dedicated to the gas phase purification, is meant to eliminate the impurities generated by material out-gassing and possible leaks, thus preventing their diffusion into the liquid.

The filling procedure of the T300 foresees the initial evacuation of air from the cryostat, the cooling down to LAr temperature to control thermal stresses and minimize out-gassing of the detector materials and the LAr filling through the liquid phase purification system.

During the test run on surface, this procedure allowed to measure an initial electron lifetime in LAr of about $100 \mu \mathrm{s}$, probably due to general out-gassing of the walls, the cables and the rest of the materials inside the volume of the chamber, during the filling. The final purification of the LAr of the T300 was performed re-circulating the Argon through the two purification systems (gas and liquid phase).

The T300 liquid phase purifier consists of a set of four 22-litre stainless steel cylinders connected in parallel, filled with a molecular sieve and an $\mathrm{O}_{2}$ absorber. It is dimensioned to allow the purification of a full T300 LAr volume starting from standard commercial LAr (concentration of $\mathrm{H}_{2} \mathrm{O}$ and $\mathrm{O}_{2}$ of about 0.5 ppm, corresponding to 120 litres of $\mathrm{O}_{2} \mathrm{NTP}$ ).

The molecular sieve, HYDROSORB ${ }^{\mathrm{TM}, 5}$ is of the $5 \mathrm{~A}$ type and occupies a volume of about 7 litres of each cylinder of the purifier; it absorbs mostly water molecules. The absorption capacity depends on the temperature and partial pressure of the water vapour; it has been measured that one litre of molecular sieve can absorb more than 20 litres of water vapour before saturation; molecular sieves are also effective against other polar molecules.

The $\mathrm{O}_{2}$ absorber, 15 litres of volume in each of the four cylinders of the purifier, is the OXISORB ${ }^{\mathrm{TM}, 6}$ : it consists of chromium oxide deposited on a $\mathrm{SiO}_{2}$ support. One litre of OXISORB can absorb up to 3.2 litres of $\mathrm{O}_{2}$ NTP; hence we can count on a total absorption capacity of about 190 litres of $\mathrm{O}_{2}$ at NTP for the full T300 liquid purifier.

It should be noticed that the molecular sieve is located upstream in the filter, because the OXISORB is less effective in the presence of water vapour. The purification efficiency has been measured to be insensitive to the LAr flux rates up to more that 1000 litre/hour [2]. The nominal recirculation speed for the T300 is set at 2000 litre/hour, i.e. 500 litre/hour for each of the four purifier cylinders; at this flux rate, the pressure drop across the filters is about 1 bar.

The liquid recirculation is obtained with the use of a cryogenic pump, immersed in an independent Dewar located on a side of the T300 cryostat. The pump is dimensioned to sustain the required recirculation speed and pressure drop. The LAr in the pump cryostat is kept at the same level and pressure as those in the detector cryostat with the help of a connection between the two gas phases. The LAr in the T300 cryostat is extracted on one side close to the liquid surface; the pump pushes

\footnotetext{
${ }^{5}$ HYDROSORB is a Trademark of MESSER Group. See data Sheet catalogue: http://www.messergroup.com/de/Produkte_und_Loesungen/Fachbroschueren/Spezialgase/Gas_purification_systems_for_the_highes _level_of_purity.pdf.

${ }^{6}$ OXISORB is Trademark of MESSER Group.
} 


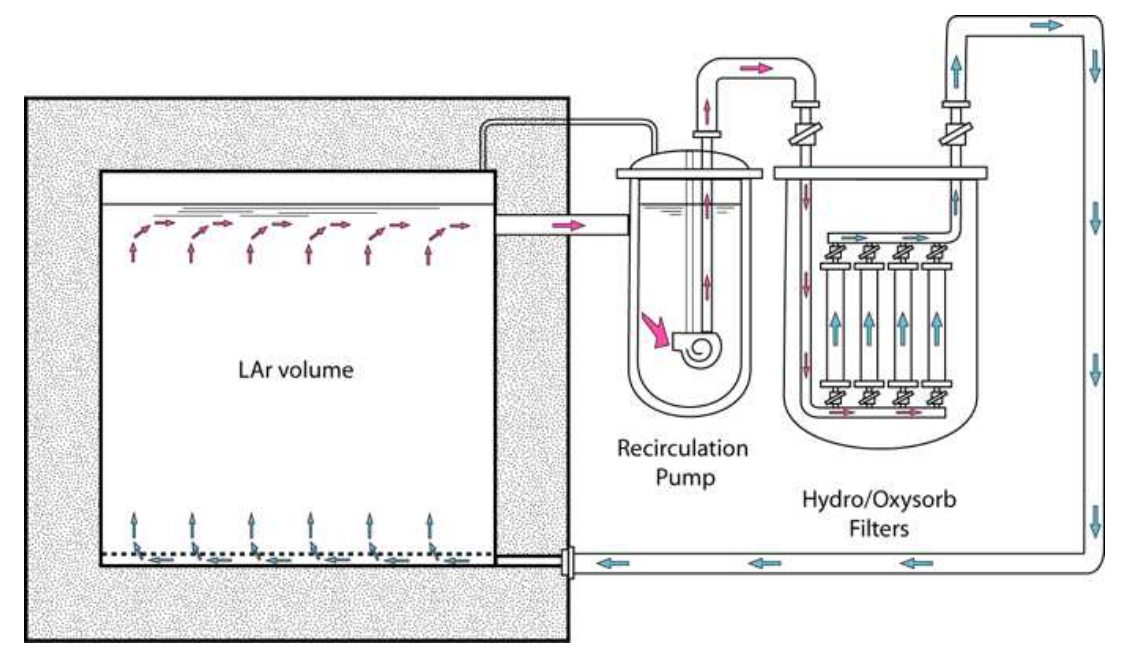

Figure 5. Schematics of the SLICE liquid phase purifier.

the LAr into the purifier, and the purified liquid is sent back to the opposite side of the cryostat, close to the floor, away from the input of the pump, in order to maximize the recirculation efficiency.

Experience with the T300 module has shown that the LAr purity increases exponentially with a mean time of about 5 days, corresponding to the time needed to re-circulate one full volume. Moreover, the purity was found to be homogeneous through all the active volume of the detector, indicating that the LAr mixing due to convective motions is much faster than the recirculation time [ [ ]. However, at the beginning of the recirculation process, the purity increases slower than expected, due to the residual out-gassing of the detector materials. This process disappears with time and a purity corresponding to an electron lifetime of several milliseconds can be reached.

In the case of the SLICE detector, the main difference in the filling procedure consists in skipping the air evacuation phase, replacing it with repeated gas flushing, as mentioned in point 9 of the previous paragraph. In addition, in order to get a better initial LAr purity, a liquid filling in non-turbulent regime is required to avoid contaminating the ultra-pure LAr with the less clean gas phase. A possible set-up to fulfil the requirements has been sketched in previous paragraph, point 3 (figure 5).

In order to remove the impurities produced by out-gassing in the GAr phase, before they diffuse into the liquid, the LAr recirculation is complemented by a GAr recirculation/purification system. Its layout is a clone of that of the T300 module and consists of two purifier units (one is shown in figure (6) collecting the gas from the chimneys that host the cables for the wire chamber read-out, with a flow rate of $50 \mathrm{~m}^{3} \mathrm{GAr} /$ hour. The gas is re-condensed into a LN2 re-condenser, the liquid dropping into an Oxysorb filter placed below the re-condenser. The purified Argon is sent back into the LAr container just below the LAr surface.

In the MODULAr detector at least 15 sets similar to the SLICE LAr purification system are needed, corresponding to the extension from $4 \mathrm{~m}$ to $60 \mathrm{~m}$ of the $(z)$ direction of the structures, to conservatively purify the full volume without saturating the filters. For a normal recirculation operation, the same 15 sets running at $2 \mathrm{~m}^{3} /$ hour would allow the full recirculation of the volume every about 5 days. 


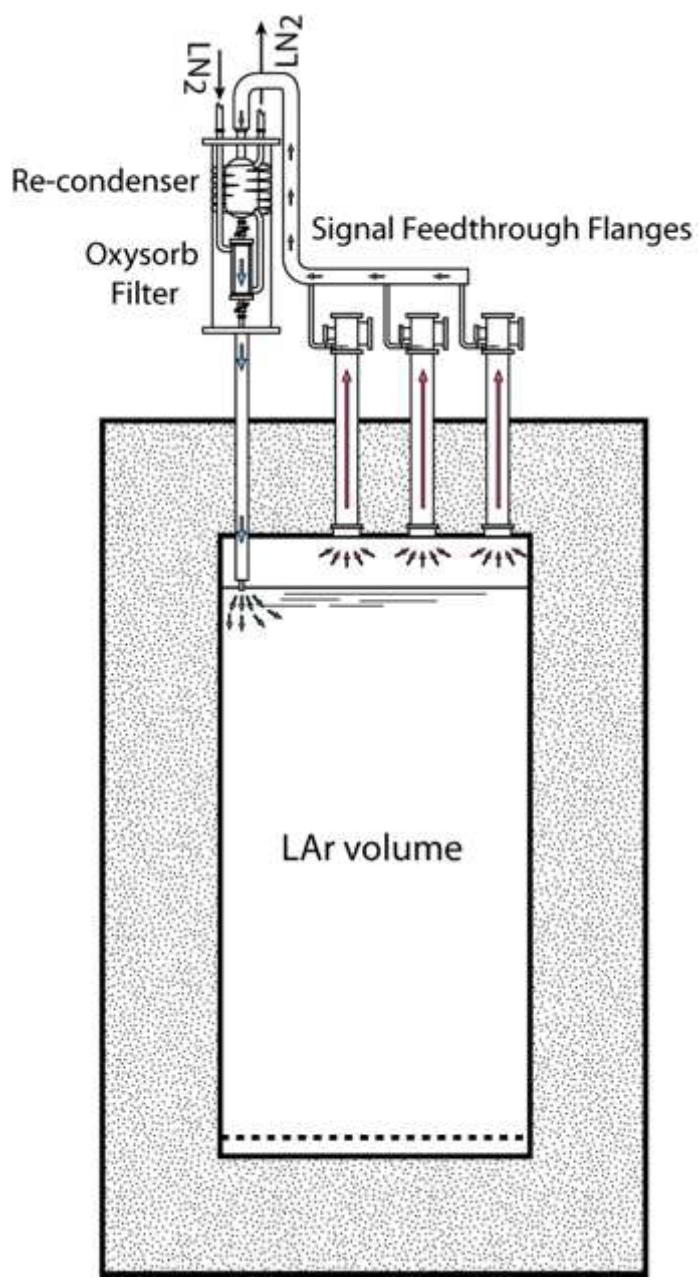

Figure 6. Schematics of one of the two gas phase recirculation system units for SLICE. The SLICE crosssection is orthogonal to that shown in figure 5.

In conclusion the SLICE realization will follow closely the experience already achieved with T300. If this experimental test is successful, the extrapolation to the full MODULAr unit appears to be straightforward.

\subsection{Considerations on air evacuation procedures for the SLICE cryostat}

The method of flushing with pure GAr is widely used already in gaseous wire and drift chambers, which are generally not evacuated. In the MODULAr case, additional problems may arise in view of the magnitude of the volume and the possible appearance of "dead" spots, in which the gas may not circulate. The SLICE test module - with its large volume and cross-section similar to the MODULAr cryostat - will be an adequate playground to perfect the method, which will be tested experimentally beforehand with smaller scale containers and carefully compared with hydrodynamic calculations.

In the idealized case of complete turbulent and continuous uniform mixing through the container, the air-GAr transition behaves exponentially with a factor $\sim 1 / \mathrm{e}$ at each passage. Therefore, 
in order to achieve attenuation of the order of $10^{-6}$, about 14 cycles are necessary. On the contrary, if the GAr is injected with little or no turbulence, for instance uniformly from the bottom with the extraction of initial air at the top, the transition GAr-air moves orderly from the bottom to the top and only pure air exits from the top, producing a faster and more orderly transition. These are ideal cases and the optimization of the initial gas purification system with pure GAr flushing and the overall filling procedure will require detailed studies with both hydrodynamic numerical simulations and measurements on appropriately scaled down vessels.

As a first preliminary step, we have set-up a bi-dimensional calculation by means of the computational fluid dynamics package Star-CD $4.02\left({ }^{\mathrm{TM}_{2}} 2006\right.$ CD-Adapco) [18] to verify the feasibility of the method.

A vertical cross-section of the SLICE detector has been considered, namely an $8 \times 8 \mathrm{~m}^{2}$ area. The GAr inlet is assumed to be at the bottom of the vessel, distributed over the full $8 \mathrm{~m}$ width (this could be obtained, for instance, with the use of several nozzles drawn in parallel from a unique inlet); the gas outflow has been assumed to proceed through the chimneys foreseen for the electronic cables (as in the ICARUS-T600). The cathode in the middle of the chamber has also been included in the simulation, as it could affect the gas flow in a sizable way.

To minimize gas mixing, the GAr flow must proceed orderly and uniformly, from bottom to top across the full $>8 \mathrm{~m}$ height of the vessel, in order to avoid possible "pockets" of limited circulation which could persist for a long time due to the slow diffusion of the gas. To this effect, a bottom containment plate with many small holes uniformly distributed over the whole surface ensures a uniform flow from bottom to top of the LAr volume. Likewise on the top, an equivalent device, placed at $\sim 50 \mathrm{~cm}$ from the vessel ceiling, has been envisaged to minimize the onset of gas streams that could create unwanted pockets of "dirty" gas. In the computational model, the containment devices have been modelled as two porous sections with infinite resistance in the horizontal direction; the gas flow is then consistently bound to proceed vertically through them.

For sake of simplicity, in this first simulation attempt no other elements of the SLICE detector have been included. Moreover, symmetry considerations allowed modelling only one half of the 2D section. Mirror symmetry has been imposed on the inner side of the computational domain, above and below the cathode wall. In this 2D simulation, the chimney diameter has been chosen such to replicate correctly the ratio between the cross-section of all chimneys and the whole detector top surface, in the 3D design. A sketch of the computational domain is reported in figure 7 .

As starting condition and to safely operate the simulation in the laminar domain, a GAr flow of about $20 \mathrm{~m}^{3} /$ hour has been chosen, corresponding to a flush cycle of 0.55 days for SLICE cryostat volume of $256 \mathrm{~m}^{3}$. In case of larger volumes, the flow could be increased proportionally.

It should be noted that given the size and the aspect ratio of the SLICE vessel, the onset of transitional effects, leading to turbulence, could show up at flow rates above $40 \mathrm{~m}^{3} /$ hour (with the Reynolds number in the chimney section exceeding $\sim 2000$ ). Nevertheless, it is worth mentioning that the effects of turbulence on the flushing procedure have not been fully investigated so far. This topic will be examined more deeply in the future, in order to establish whether a global acceleration of the process is actually achievable.

The simulation has been protracted in order to cover a time span of 6 flushing cycles. As an evidence for the laminar hypothesis, the calculated flow field approaches monotonically a steady state. A snapshot of the streamlines at steady state is portrayed in figure 7 . The stabilizing effect 


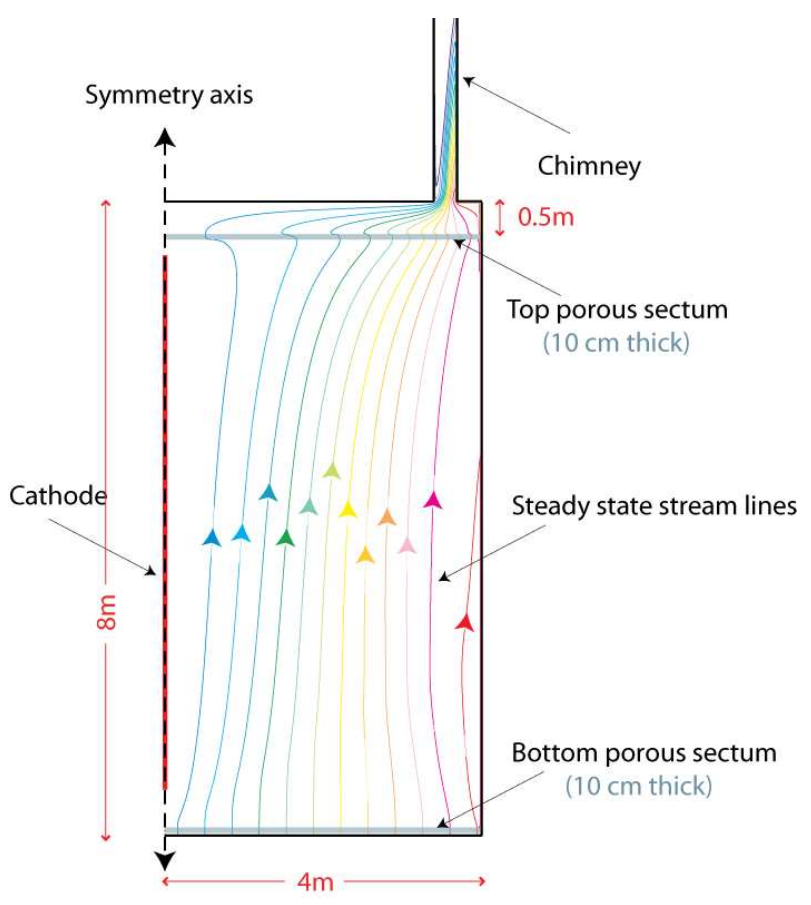

Figure 7. Sketch of the computational domain representing a SLICE cross-section. Cathode, top and bottom porous septa, electronic chimneys are included in the simulation set-up. GAr flows from the bottom to the top. The steady state stream lines of the GAr flow through the SLICE vessel are also shown. Velocities along the flow lines increase from blue to red.

of the containment plates is clearly visible: the gas stream bends orderly towards the outflow just above the upper plate, thus avoiding the onset of secondary re-circulations in the central zone of the chamber.

Such favourable flow features are reflected in the time evolution of the Ar concentration (figure 8). After 1000 seconds ( $\sim 0.02$ cycles, figure $8 \mathrm{a}$ ), the inflowing GAr is still concentrated in the lower part of the chamber. The shape of the contour bands suggests that the GAr-air mixing happens mainly by diffusion. After one cycle, at $t=13.3$ hours (figure Bb), the situation has evolved, clearly showing the effect of the flow field on the concentration of GAr. Due to the higher gas speed, the bulk of the transported GAr is found in the core region of the stream, while beside both cathode and outer wall, a diffusive gradient persists.

At $\mathrm{t}=26.6$ hours $(\sim 2$ cycles, figure $8 \mathrm{k})$ ), the flow has already reached steady state. The bulk of GAr has expanded, occupying almost the whole active part of the chamber. The completion of the flushing process is then depending on diffusive effects, as shown in figure $8 \mathrm{~d}$. In fact, after 6 cycles, the concentration field remains qualitatively unchanged, although the maximum residual concentration of air is reduced to $3 \mathrm{ppm}$.

The whole process is summarized in figure 9 , where the average air fraction in the chamber is plotted against the elapsed time. After an initial transient phase (slightly more than 1 cycle), the residual concentration drops down exponentially, reaching $1 \mathrm{ppm}$ after almost 5.5 cycles.

The results presented here indicate that the initial evacuation of air through GAr flushing is a viable solution, with an average residual impurity concentration less that $1 \mathrm{ppm}$ in gas phase 


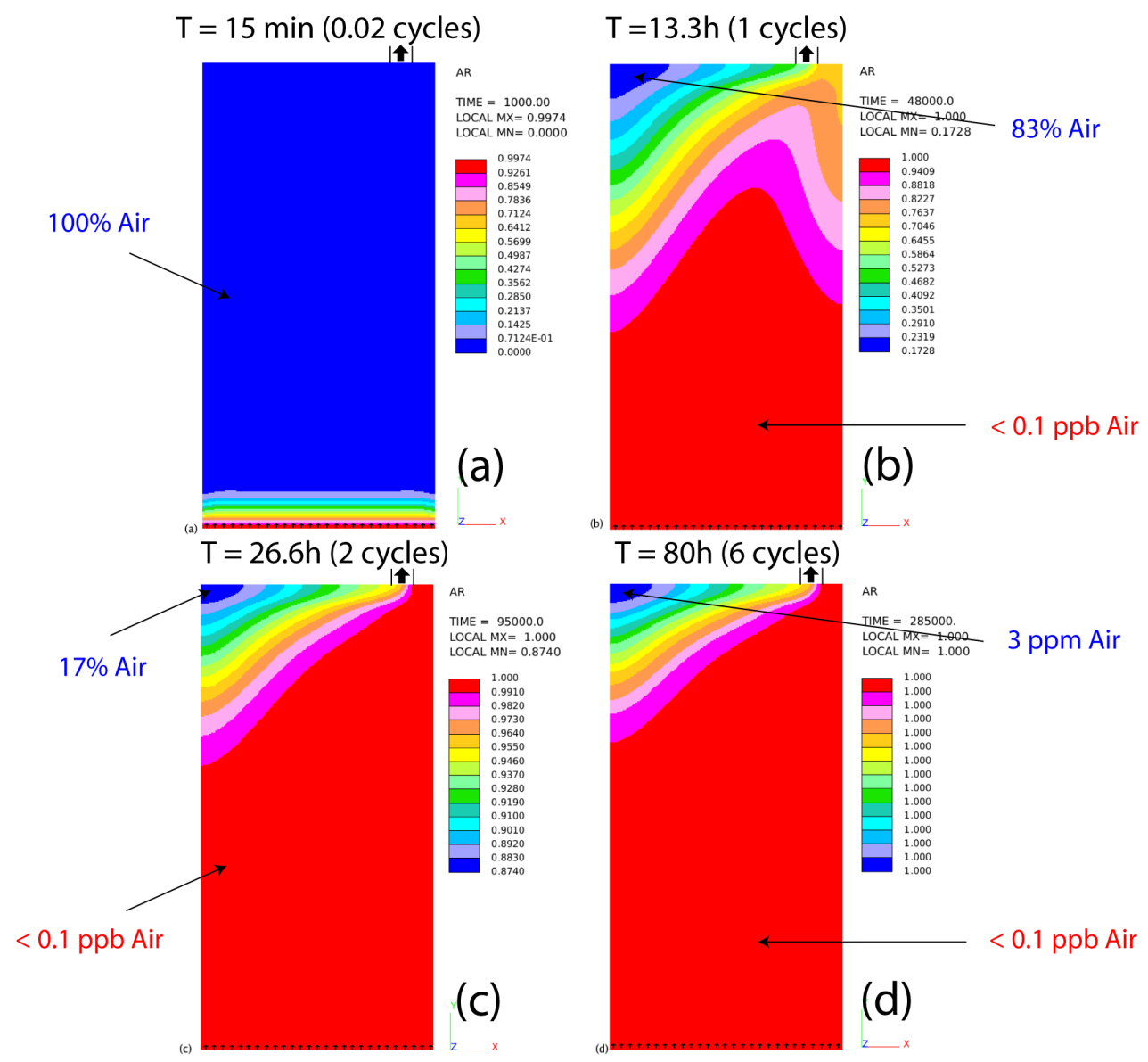

Figure 8. Time evolution of the residual air concentration in the SLICE detector. The layout is that sketched in Figure 7. After two cycles a steady state GAr flow is already reached. After that, the air concentration reduces by diffusive effects.

reached in few days. More detailed simulations, based on 3D modelling and including additional detector details are envisaged in parallel with small-scale tests.

\subsection{Implementing the DAQ of MODULAr and SLICE}

The ICARUS-T600 electronics has been described in various papers [ [ . Starting from the experience gained with the ICARUS-T600 operation, an upgraded DAQ scheme is proposed that implements the same basic architecture with new, better performing components and different modularity in view of a multi-kton LAr-TPC.

\subsubsection{The ICARUS-T600 DAQ}

The ICARUS-T600 read-out architecture was designed for a detector comprising $510^{4}$ channels. The analogue chain consists of a front-end low noise charge sensitive pre-amplifier, based on a custom designed BiCMOS dual channel IC with a j-FET input stage, followed by a baseline restorer to reduce low frequency noise. The gain of the front-end amplifier and filter is $1 \mathrm{~V} / 164 \mathrm{fC}$. The 10-bit ADC input range is $1 \mathrm{~V}$, and the least count is equivalent to 1000 electrons. This value is 


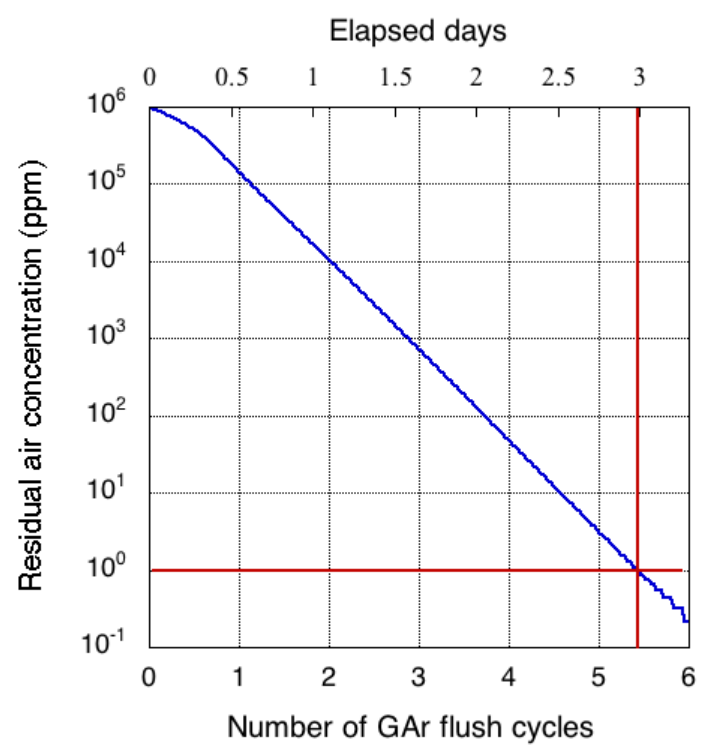

Figure 9. Residual air concentration in SLICE, averaged over the whole vessel volume, as a function of the number of GAr flushing cycles (blue curve). Also reported is the number of elapsed days. The Argon gas flow rate is $20 \mathrm{~m}^{3} / \mathrm{h}$. The SLICE volume is $256 \mathrm{~m}^{3}$. The level of $1 \mathrm{ppm}$ is reached in about 3 days.

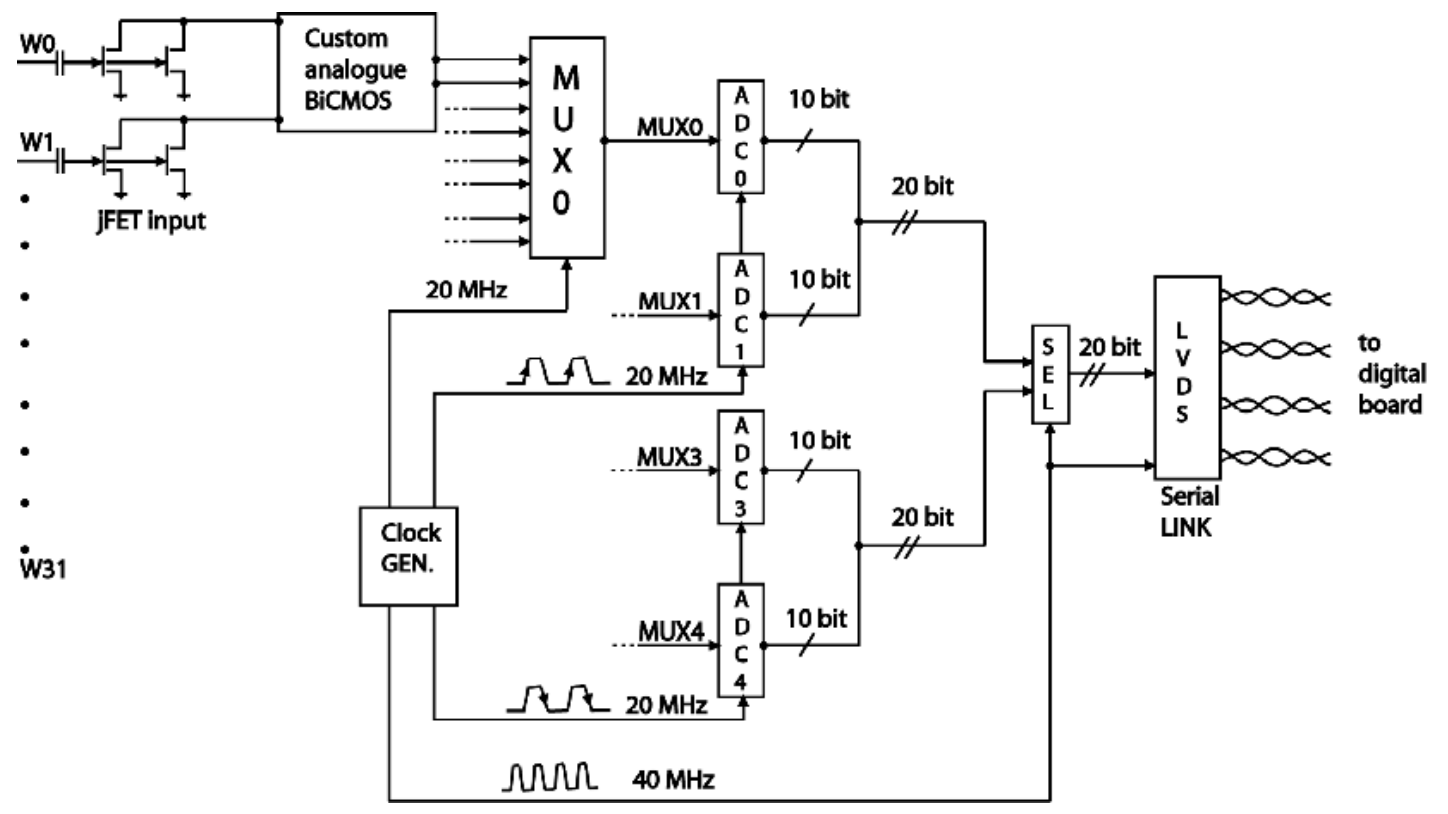

Figure 10. Block diagram of the ICARUS-T600 analogue board (CAEN V789).

comparable to the amplifier noise of $\sim 1200$ electrons at detector capacitance of $450 \mathrm{pF}$ (signal wire plus cables).

The basic modularity of the system is 32 . The digitizing stage consists in a set of 32 channels connected to four 8:1 multiplexer, each one followed by a 10-bit ADC sampling at $20 \mathrm{MHz}$. The sampling time is $400 \mathrm{~ns}$ per channel. For each group of two ADC's the two 10-bit outputs are merged in one 20-bit word. As the positive clock edge drives one group of two ADC's and the 

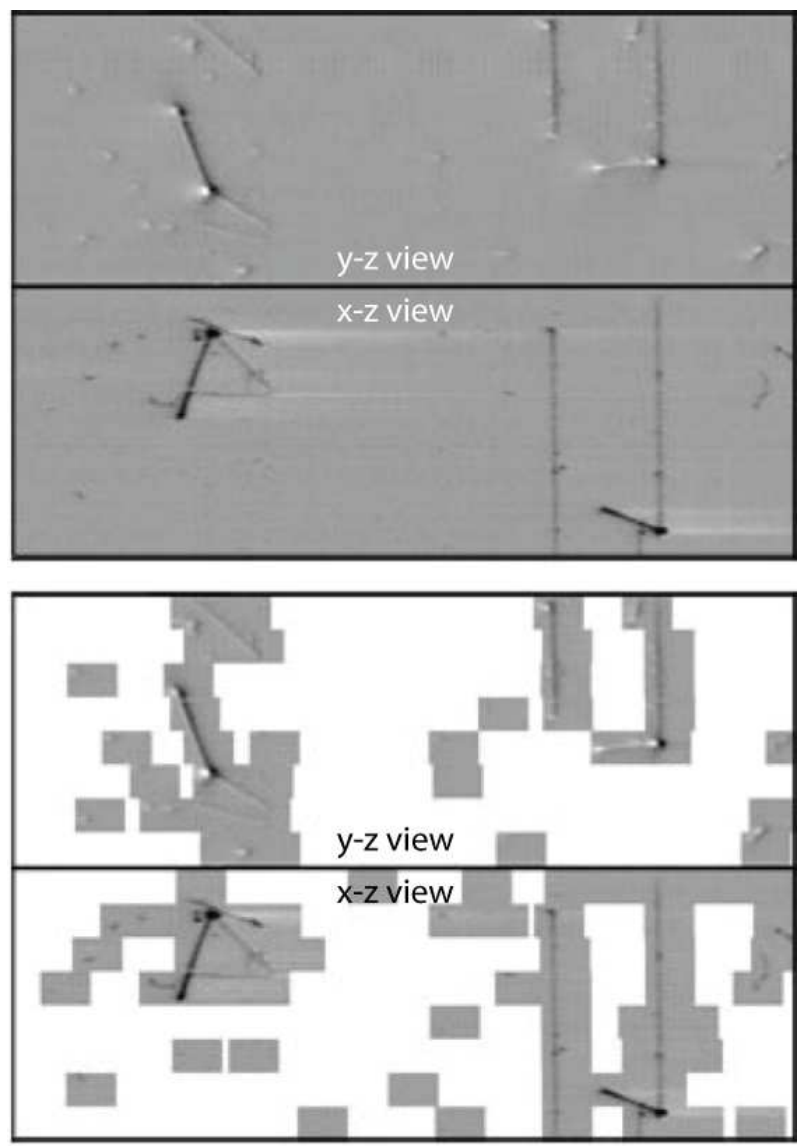

Figure 11. Example of data reduction performed by the DAEDALUS chip on one LAr-TPC event; two orthogonal views are shown. Top: full drift no data reduction; bottom: reduced data set.

negative clock edge drives the other group of two, 20-bit words can be transmitted at $40 \mathrm{MHz}$ rate on single 20-bit wide channel. This scheme is implemented on a single VME-like analogue board (CAEN V789) hosting 32 channels amplifiers, multiplexers, ADC's and a 20-bit, $40 \mathrm{MHz}$ serial link that sends the data to a digital board (CAEN V791) for the buffering of events. The serial link is LVDS (Low Voltage Differential Signal) and serializes the 20-bit data and clock on four copper lines with an equivalent data throughput of $800 \mathrm{Mb} / \mathrm{s}$. The block diagram is in figure 10 .

The receiving digital board, named ARIANNA, hosts two custom-made feature extraction ASIC chips (DAEDALUS) for hit finding, zero skipping and self-triggering. Each DAEDALUS chip operates on the data stream of a set of 16 channels and controls also the circular memory buffer. DAEDALUS includes also a median filter to reduce high frequency noise. A 28-bit absolute time register is associated to each data buffer in the memory to allow alignment of data in the event reconstruction. The board complies with the VME electrical and mechanical standard. As an example, the data reduction performed by DAEDALUS on one LAr-TPC event is shown in figure 11 [ $₫$ ].

\subsubsection{Critical issues}

The DAQ architecture for ICARUS-T600 was conceived in 1997 and DAEDALUS and front-end dual channel BiCMOS circuits were designed in 1998. The full system $(\sim 50,000$ channels $)$ 


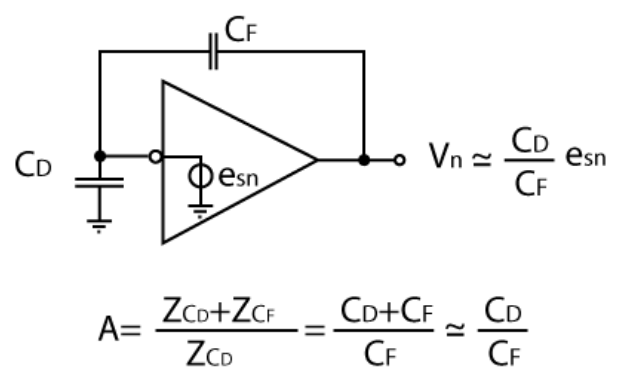

Figure 12. Equivalent circuit for noise propagation in a charge sensitive amplifier, as used in the ICARUST600 front-end.

was designed and built from 1999 to 2000 . Today it would not be possible to replicate the same circuits, because many basic components are now discontinued. In view of the scaling up of the ICARUS DAQ scheme to fit the MODULAr requirements in term of channel number $\left(>10^{5}\right)$ and cost (aiming at 60 Euros/channel), an in-depth revision of the design must take into account the new components available on the market.

Today, one evident limitation of the ICARUS-T600 DAQ is due to the use of the VME standard for the digital boards. For the analogue boards the same $6 \mathrm{U}$ Eurocard standard was adopted, with a custom backplane to connect the inputs from wires and distribute common signals (ADC baseline bias, enable signals, test pulses, etc.).

In the ICARUS-T600 DAQ 18 ARIANNA boards are housed in one VME crate that serves a total of 576 channels. One crate is connected to an analogue crate with the same modularity, which in turn receives the signals from a single ICARUS-T600 flange that has 18 feed-through connectors, one for each set of 32 channels. Configuration and control of the 18 boards relies on a dedicated VME CPU, which also handles the data transfers from board buffers to the Ethernet event builder network. Performance of the DAQ system is bounded by the VME throughput ( $8-10 \mathrm{MB} / \mathrm{s}$ ) of the slave ARIANNA board. The sequential access to the ARIANNA boards in each crate, inherent to the shared bus architecture, limits furthermore the overall throughput.

The redesign of the system can overcome this limitation adopting a modern switched I/O, as PCI Express standard, allowing the parallelization of the data flows. In addition, such I/O transaction can be carried over low cost optical Gb/s serial links. This allows a more effective modularity of the digital hardware architecture, decoupled from the geographical distribution of the signal feed-through's, thus leading to a larger integration and a lower cost per channel.

\subsubsection{The amplifier}

The capacitance associated to each channel is determined by the capacitance of the wires, of the order of $20 \mathrm{pF} / \mathrm{m}$, in parallel with the capacitance of the cable, of the order $50 \mathrm{pF} / \mathrm{m}$. In the following, an input capacitance of $\sim 600 \mathrm{pF}$ is assumed as a reasonable value for a 10 m electrode wire, $6 \mathrm{~mm}$ pitch, and an average $8 \mathrm{~m}$ of connecting cable per wire.

The dominant noise in a high capacitance detector [20] is the series noise $\mathrm{e}_{s n}$ (voltage noise) linearly increasing with the input total capacitance $\left(\mathrm{C}_{D}\right)$ while the parallel noise (current noise) contribution is proportional to the shaping time of the signal. 


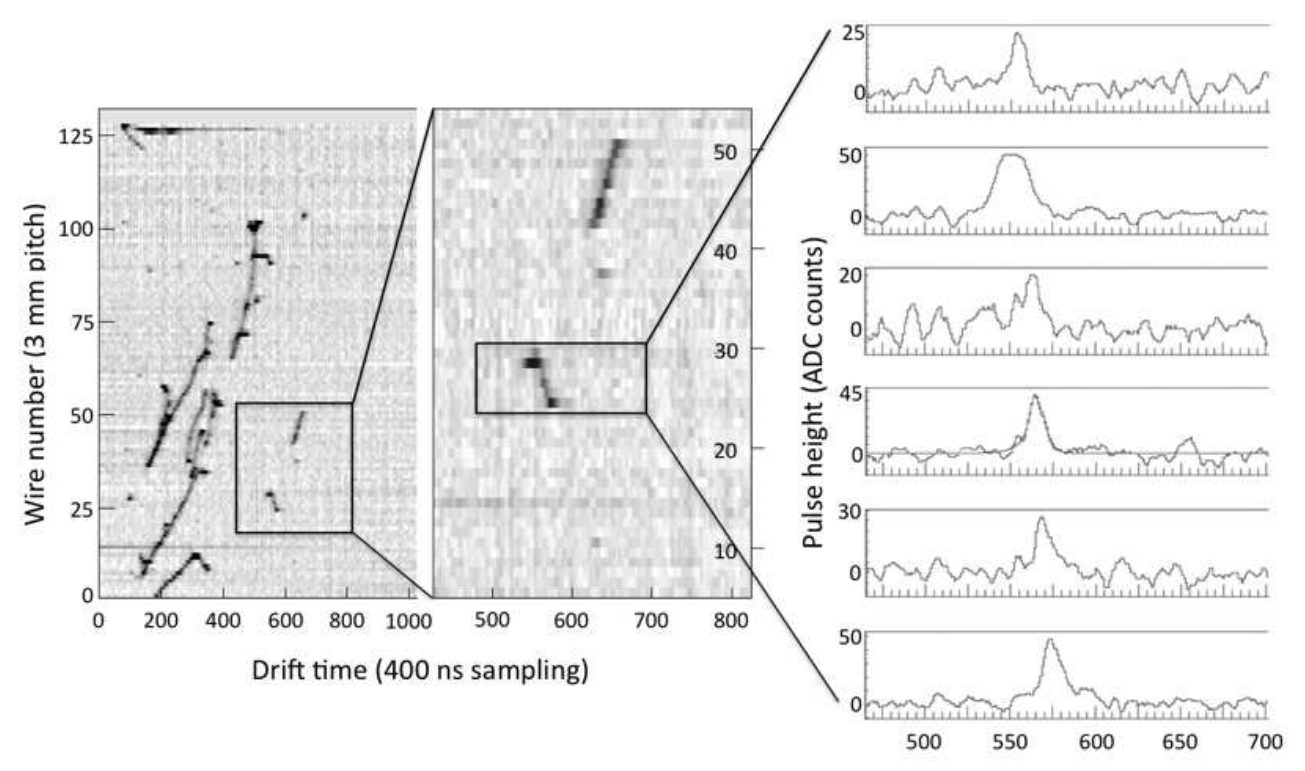

Figure 13. Left: Image of a low energy electromagnetic shower in a LAr-TPC. Drift time is along horizontal axis (400 ns sampling). Wire numbering is on the vertical axis ( $3 \mathrm{~mm}$ pitch). Middle: Zoom of the selected area on the left image. Right: Waveforms of the wire signals in the selection box on the middle image, showing the typical signal shapes of minimum ionizing particles.

The equivalent circuit showing the effect of the input capacitance on the serial noise is sketched in figure 12. The serial noise generator has a noise density, $\mathrm{e}_{s n}^{2} \propto \mathrm{g}_{m}^{-1}$, where $\mathrm{g}_{m}$ is the transconductance of the input device. It is evident that a very high transconductance active device in the first stage of the amplifier is necessary to minimize the effect of the $\mathrm{C}_{D}$. In fact the signal to noise ratio is given by $S / N \propto q /\left(e_{S n} \cdot C_{D}\right)$, where q is the detector signal in terms of collected charge.

The best component would be the bipolar transistor that exhibits transconductance of the order of $\mathrm{g}_{m} \sim 400 \mathrm{mS}$ at $\mathrm{I}_{c}=100 \mathrm{~mA}$ with an amplification merit factor $\left(\mathrm{g}_{m *} \mathrm{Z}_{\text {out }}\right)$ higher than 3-4 10 $0^{5}$. A second option would be the j-FET transistor that has a much lower transconductance, of the order of $\mathrm{g}_{m} \sim 40 \mathrm{mS}$ at $\mathrm{I}_{c}=10 \mathrm{~mA}$ with a merit factor around 3-4 $10^{3}$. We do not consider CMOS because of the extremely low transconductance and unfavourable amplification merit factor. The bipolar transistor has a typical parallel noise density $\mathrm{i}_{p}=1-2 \mathrm{pA} / \sqrt{ } \mathrm{Hz}$ and gives an unsustainable noise contribution. Therefore, the $\mathrm{j}$-FET, with a reasonable transconductance, a negligible parallel noise $\left(\mathrm{i}_{p}=0.001 \mathrm{pA} / \sqrt{ } \mathrm{Hz}\right)$ and a serial noise density of about $1-2 \mathrm{nV} / \sqrt{ } \mathrm{Hz}$, is the best choice.

In LAr a minimum ionizing particle releases a charge of $2 \mathrm{fC} /$ wire for a $3 \mathrm{~mm}$ pitch. The ICARUS amplifier, designed with a j-FET input, has an Equivalent Noise Charge (ENC) of $\sim 1250$ electrons at $\mathrm{C}_{D}=450 \mathrm{pF}$. In figure 13-left an image of a LAr-TPC event is shown as an example. In figure 13-right signal waveforms from adjacent wires, belonging to the short track in the box, are evidenced. The average signal is 12 ADC counts for an r.m.s. noise of 1.2 counts corresponding to a $\mathrm{S} / \mathrm{N} \sim 10$. In the case of MODULAr, the long wire capacitance, including the cable contribution, should not exceed $900 \mathrm{pF}$. Given the $6 \mathrm{~mm}$ wire pitch, the foreseen electronics should provide a $\mathrm{S} / \mathrm{N}$ ratio of $\sim 10$ for the long horizontal wires. For the short stereo wires (with a total capacitance of $600 \mathrm{pF}$ ) the $\mathrm{S} / \mathrm{N}$ should be even better. 


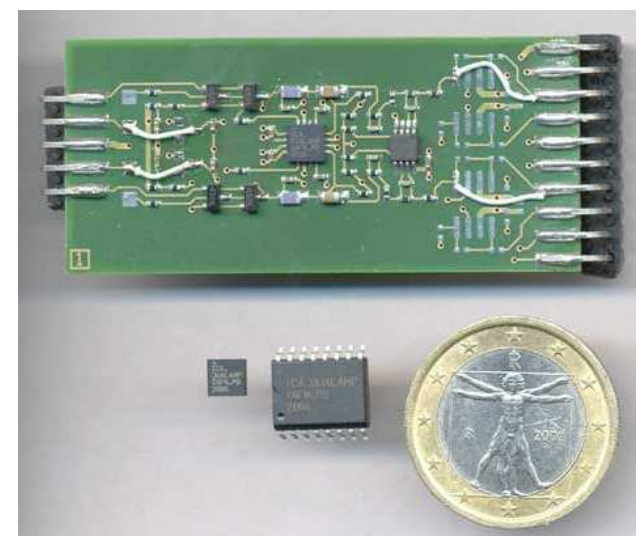

Figure 14. Top: a prototype of a four channel amplifier and filter designed with the IC in the new package. Bottom left: the new package compared with the original used in T600 (bottom centre).
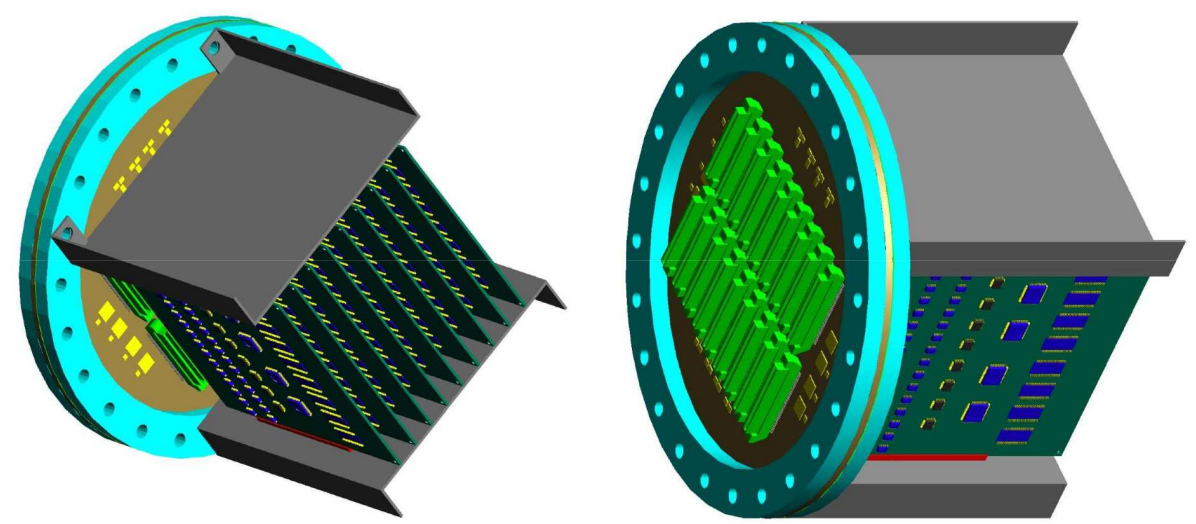

Figure 15. Flange for 576 channels equipped with amplifiers.

The custom IC [21] adopts an unfolded cascode architecture for the integrator realized in BiCMOS technology, with external j-FET's at the input. Two discrete j-FET's are connected in parallel to reach a total $\mathrm{g}_{m}$ of the order of $50-60 \mathrm{mS}$, at a reasonable bias current.

Two identical channels have been integrated on each IC. This choice allows designing an absolutely symmetrical layout for the two channels obtaining identical electrical behaviour.

A revisited version of the ICARUS front-end amplifier IC has been developed, based on the present design, but realized in a much smaller package $\left(4 \times 4 \mathrm{~mm}^{2}\right.$, see figure 14). This package will allow a higher degree of integration. A relevant number of components is already available for prototyping and about $1.410^{5}$ dies on sealed silicon wafers are ready.

This front-end layout can be hosted in a compact crate very close to the feed-through flange. Alternatively, the use of the flange itself as a backplane supporting the analogue boards could be envisaged. This solution is under study; a possible layout for 576 channels is shown in figure 15; the boards that host amplifiers, as the one in figure 14, are also shown. The number of connectors and cables would be drastically reduced with a benefit for cost and $\mathrm{S} / \mathrm{N}$ value.

The number of channels served by one feed-through flange (576 in the proposed layout) will define the new modularity. That means that the new analogue module will be either the crate just 
described or the flange with boards. This new 576 channels module, replacing the old analogue board (modulo 32), will also perform the data conversion before transmitting data to the digital buffering board that will now be discussed.

The solution of installing the front-end amplifiers close to the wires inside the detector was seriously considered but eventually discarded for the following reasons. The only active component with a reasonable trans-conductance at liquid Argon temperature is the n-type j-Fet. With this technology the integration of complex components is rather difficult, not to mention the cost of this construction process. Moreover large biasing currents (leading to large heat dissipation in LAr, several $\mathrm{mW} / \mathrm{channel}$ ) are needed to reach the required trans-conductance values of 20-60 mS.

It is worth mentioning that, during the R\&D phase of ICARUS-T600, a j-Fet integrated preamplifier working at LAr temperature was designed. Prototypes were successfully tested inside a small LAr-TPC (256 channels) showing comparable performance with respect to the external read-out. However, in the opinion of the collaboration, this solution is not suitable for large volume detectors, also because the inaccessibility of the front-end for maintenance and repair is a major and serious drawback.

\subsubsection{The AD conversion}

A wide range of suitable serial ADC's, converting the data as a sequence of bits at high rate, is already available on the market. The data rate of the serial bits is typically around 10-12 times higher than the expected sampling frequency; to reach $3 \mathrm{MHz}$ sampling rate with 12-bit resolution, these ADC's must be clocked at $48 \mathrm{MHz}$.

This class of devices is quite interesting in terms of cost, power consumption and dimensions. Typically, they are packed in Mini Small Outline Package (MSOP) smaller than $5 \times 5 \mathrm{~mm}^{2}$. The acceptable sampling frequency for a TPC with $6 \mathrm{~mm}$ pitch can be assumed in the range of 11.5 $\mathrm{MHz}$ for which there is already a wide choice of devices. We can assume a resolution of 10-bit but also 12-bit ADC's are available. The arrangement of the ADC's after the amplification is shown in figure 16.

The use of a set of a few FPGA for 576 channels handles, filters, and organizes the serial information. Assuming a 10-bit ADC's, sampling at a frequency of $1.5 \mathrm{MHz}$, and data compression in one byte, a transmission rate of $6912 \mathrm{Mb} / \mathrm{s}$ is required. On an optical fibre, balancing zeros and ones, it becomes $8640 \mathrm{Mb} / \mathrm{s}$. Optical links with $1.5 \mathrm{~Gb} / \mathrm{s}$ data rates are standard and can be driven by the RocketIO ${ }^{\mathrm{TM}}$ interfaces available on many FPGA's from different vendors. Six optical links could serve all the channels of one module (576 channels) and convey also extra information, as absolute time. Some of the links will be bi-directional to distribute absolute clock and simple commands. The ADC's and FPGA's will be housed in the same crate next to the flange or even on special boards on top of the amplifiers boards shown in figure 14.

The implementation of the equivalent of the ICARUS-ARIANNA board is not discussed here. One could say that, nowadays, ASIC VLSI will not be required for hit finding, as the entire feature extraction algorithm can be implemented in powerful FPGA.

\section{Conclusions}

The forthcoming operation of the ICARUS-T600 detector in the real experiment CNGS2 will rep- 


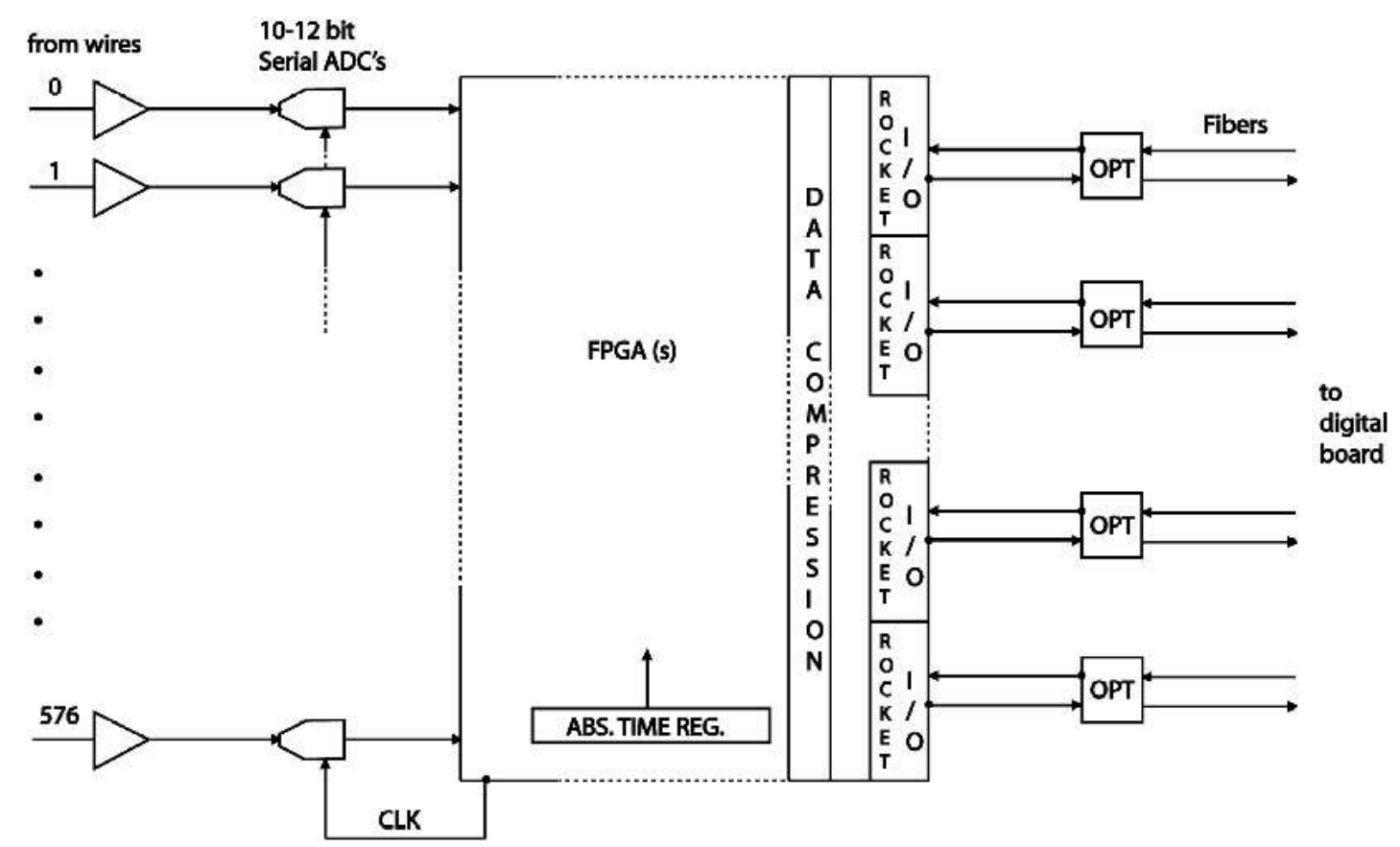

Figure 16. Block diagram of the upgraded DAQ system including the Serial ADC's.

resent the completion of a development of the LAr-TPC chamber over more than two decades. As it has been described in this paper, the operation of the ICARUS-T600 evidences that a number of important milestones have been already achieved in the last several years, opening the way to the development of a new line of modular elements of $5 \mathrm{kt}$ each and which can be progressively extrapolated to the largest conceivable LAr-TPC sensitive masses, for the study of accelerator and non accelerator driven phenomena.

The new MODULAr detector will maintain the majority of components we have already developed, in particular:

- The readout electronics and the data acquisition of the ICARUS-T600. The 50'000 channels of electronics already at hand are adequate for about 10'000 tons of sensitive mass of new modular elements. Nevertheless a new design of the readout electronics, based on modern components, requires limited $\mathrm{R} \& \mathrm{D}$ and is under development.

- The signal feed-through's for a very large number of signal wires, developed with a new technology patented by INFN/Padova. They are extremely reliable and capable of ensuring adequate high vacuum tightness for many tens of thousands channels at LAr temperature.

- An original and extremely robust technology for the readout wires, capable of withstanding the wide variations in temperature (-200 K) in the cooling and warm up phases. This technology has been developed for ICARUS-T600 in collaboration with industry. So far, not a single wire has broken in the ICARUS-T600 detector, in spite of the many operations and of the transport on road for about $600 \mathrm{~km}$. Although the wires are about 2.66 times longer, the same method will be cloned to the new modular elements. The realization is simple, fast and cheap and is realized with the help of an automatic machine. 
- The high voltage feed-through's and the appropriate noise filtering $(>100 \mathrm{~dB})$ to remove the electric pick-up from the very small signals of the wire chambers, developed and fully operated with no problem at $150 \mathrm{kV}$, twice the design voltage of the ICARUS-T600. The design voltage of the new modular elements is $200 \mathrm{kV}$.

- The LAr purification and re-circulation systems. The purity of the LAr is generally well below the required level of $310^{-10}$ of equivalent Oxygen purity, which has required a dedicated technological development over many years. The LAr is continuously purified both in the gas and in the liquid phase and circulated with adequate low temperature pumps. The purification system can be expanded in a straightforward way to become adequate to the new modular elements. A great deal of experience has taught us how to remove materials that are producing significant leakage.

- Photomultipliers designed to ensure a appropriate photocathode efficiency at the LAr temperature and equipped with a thin deposit of wavelength shifter (TPB) on the photo-cathode, to convert the $128 \mathrm{~nm}$ Argon light into the visible.

- An appropriate method of high precision purity monitors, in order to monitor in real time the purity of the LAr.

As already pointed out, the main domain of remaining developments, to ensure the correct realization of the new modular elements, is related to the streamlining and simplification of the mechanical structures, to the reduction of the overall costs and to the new developments, previously described, of the structure of the detector, which are:

- The use of perlite for the cryogenic structure. Perlite is vastly in use in the cryogenic industry and should represent no problem.

- The filling process starting from air to pure LAr, taking into account the motion of the gas, optimizing the inlet and outlet geometries and minimizing the number of cycles.

- The thermal convections of the LAr, in order to optimize the temperature gradients and to insure a convincing circulation in all regions of the dewar, both in the cool down phase and in the stationary state.

- The out-gassing rate and the recirculation processes required in order to achieve the required electron lifetime.

- The geometry of the compact re-circulators, both in the liquid and in the gaseous phases.

The realization of these developments should not face any major problem and the detailed engineering design of the first new modular elements should proceed smoothly and rapidly.

The exposure of a $20 \mathrm{kt}$ fiducial mass to a new neutrino beam $10 \mathrm{~km}$ off-axis will allow exploring with a largely improved sensitivity the $v_{\mu} \leftrightarrow v_{e}$ oscillations [8].

Finally, the realization of the SLICE test unit with the same cross-section but reduced length and mass of the MODULAr unit, as intermediate step, will also allow carrying out an interesting experimental program in neutrino physics, investigating the existence of sterile neutrinos and measuring neutrino cross-section in the $0-3 \mathrm{GeV}$ energy range. 


\section{References}

[1] C. Rubbia, The Liquid-Argon Time Projection Chamber: A New Concept For Neutrino Detector, CERN-EP/77-08 (1977).

[2] S. Bonetti et. al., A study of the electron image due to ionizing events in a two-dimensional liquid argon TPC with a 24 cm drift gap, Nucl. Instrum. Meth. A 286 (1990) 135;

A. Bettini et. al., A study of the factors affecting the electron lifetime in ultrapure liquid argon, Nucl. Instrum. Meth. A 305 (1991) 177;

P. Benetti et. al., A 3-ton liquid argon time projection chamber, Nucl. Instrum. Meth. A 332 (1993)

395; Argon purification in the liquid phase, Nucl. Instrum. Meth. A 333 (1993) 567;

P. Cennini et. al., Performance of a 3-ton liquid argon time projection chamber, Nucl. Instrum. Meth. A 345 (1994) 230;

P. Benetti et. al., A $3-D$ image chamber for the LAr TPC on multilayer printed circuit board, Nucl. Instrum. Meth. A 346 (1994) 550;

P. Cennini et. al., Improving the performance of the liquid argon TPC by doping with tetramethyl germanium, Nucl. Instrum. Meth. A 355 (1995) 660; A neural network approach for the TPC signal processing, Nucl. Instrum. Meth. A 356 (1995) 507;

F. Arneodo et. al., Performance evaluation of a hit finding algorithm for the ICARUS detector, Nucl. Instrum. Meth. A 412 (1998) 440;

F. Arneodo et. al., The ICARUS 50-l LAr TPC in the CERN nu beam, hep-ex/9812006,

P. Cennini et. al., Detection of scintillation light in coincidence with ionizing tracks in a liquid argon time projection chamber, Nucl. Instrum. Meth. A 432 (1999) 240,

F. Arneodo et. al., First observation of 140-cm drift ionizing tracks in the ICARUS liquid-argon TPC, Nucl. Instrum. Meth. A 449 (2000) 36;

F. Arneodo et. al., Determination of through-going tracks' direction by means of delta-rays in the ICARUS liquid argon time projection chamber, Nucl. Instrum. Meth. A 449(2000) 42, Performance of the $10 \mathrm{~m}^{3}$ ICARUS liquid argon prototype, Nucl. Instrum. Meth. A 498 (2003) 292.

[3] G. Aquistapace et al., The CERN neutrino beam to Gran Sasso (NGS), CERN-98-02,

INFN-AE-98-05 (1998);

R. Baldy et al., The CERN neutrino beam to Gran Sasso, Addendum, CERN-SL-99-034-DI, INFN-AE-99-05 (1999);

A. Ferrari et al., CNGS neutrino beam: from CERN to Gran Sasso, Nucl. Phys. B Proc. Suppl. 168 (2007) 169.

[4] S. Amerio et al., Design, construction and tests of the ICARUS T600 detector, Nucl. Instrum. Meth. A 527 (2004) 329;

F. Arneodo et. al., Observation of long ionizing tracks with the ICARUS T600 first half-module, Nucl. Instrum. Meth. A 508 (2003) 287;

S. Amoruso et. al., Analysis of the liquid argon purity in the ICARUS T600 TPC, Nucl. Instrum. Meth. A 516 (2004) 68;

M. Antonello et. al., Detection of Cherenkov light emission in liquid argon, Nucl. Instrum. Meth. A 516 (2004) 348;

S. Amoruso et. al., Study of electron recombination in liquid argon with the ICARUS TPC, Nucl. Instrum. Meth. A 523 (2004) 275; Measurement of the mu decay spectrum with the ICARUS liquid argon TPC, Eur. Phys. J. C 33 (2004) 233;

A. Ankowski et. al., Characterization of ETL 9357FLA photomultiplier tubes for cryogenic temperature applications, Nucl. Instrum. Meth. A 556 (2006) 146;

A. Ankowski et. al., Measurement of through-going particle momentum by means of multiple scattering with the ICARUS T600 TPC, Eur. Phys. J. C 48 (2006) 667; 
ICARUS-MILANO collaboration, F. Arneodo et. al., Performance of a liquid argon time projection chamber exposed to the CERN West Area Neutrino Facility neutrino beam, Phys. Rev. D 74 (2006) 112001 .

[5] M. Baldo-Ceolin et al., ICARUS I: an optimized, real-time detector of solar neutrinos, Experiment proposal, LNF-89-005-R (1989);

P. Cennini et al., ICARUS II: second generation proton decay experiment and neutrino observatory at the Gran Sasso laboratory (Volume I), Experiment proposal, LNGS-94-99-I (1993); ICARUS II: second generation proton decay experiment and neutrino observatory at the Gran Sasso laboratory (Volume II), Experiment proposal, LNGS-94-99-II (1994); A first 600 ton ICARUS detector installed at the Gran Sasso laboratory, Addendum to proposal, LNGS-95/10 (1995); A search programme for explicit neutrino oscillations at long and medium baselines with the ICARUS detector, Experiment proposal, CERN-SPSLC-96-58, SPSLC-P304 (1996); ICARUS-Like technology for long baseline neutrino oscillations, Experiment proposal, CERN-SPSC-98-33 (1998);

F. Arneodo et al., ICANOE: a proposal for a CERN-GS long baseline and atmospheric neutrino oscillation experiment, Experiment proposal, INFN-AE-99-17, CERN-SPSC-99-25, SPSC-P314 (1999); ICANOE: preliminary techincal design and cost estimate, Addendum to proposal, LNGS-99-21-ADD-1, CERN-SPSC-99-39, SPSC-P-314-ADD-1 (1999); ICANOE: answers to questions and remarks concerning the ICANOE project, Addendum to proposal, LNGS-99-21-ADD-2, CERN-SPSC-99-40, SPSC-P-314-add-2 (1999); THE ICARUS EXPERIMENT: a second generation proton decay experiment and neutrino observatory at the Gran Sasso, Initial Physics Program, Experiment proposal, LNGS-P28/2001, LNGS-EXP-89-13-ADD-1 (2001); THE ICARUS EXPERIMENT. Addendum: cloning of T600 modules to reach the design sensitive mass, Addendum to proposal, CERN-SPSC-2002-027, SPSC-P-323

LNGS-EXP-89-13-ADD-2 (2001); A Magnetized Muon Spectrometer For ICARUS T3000 At The LNGS/CNGS, Addendum to proposal, CERN-SPSC-2003-030, LNGS-EXP-89-13-ADD-3, SPSC-P-323 Add.1 (2003).

[6] THE T2K collaboration, Y. Itow et. al., The JHF-Kamioka neutrino project, hep-ex/0106019; T. Kobayashi, Super muon-neutrino beams: physics reach and open questions, J. Phys. G 29 (2003) 1493 ;

K. Nishikawa, Long baseline neutrino experiment in Japan, in Proceedings of Third International Workshop on NO-VE: Neutrino Oscillations in Venice, Venice, Italy, 7-10 February 2006, pg. 181-194.

[7] NOvA collaboration, D.S. Ayres et. al., NOvA proposal to build a 30-kiloton off-axis detector to study neutrino oscillations in the Fermilab NuMI beamline, hep-ex/0503053.

[8] B. Baibussinov et al., A new, very massive modular Liquid Argon Imaging Chamber to detect low energy off-axis neutrinos from the CNGS beam (Project MODULAr), Astropart. Phys. 29 (2008) 174.

[9] A. Ereditato and A. Rubbia, The liquid Argon TPC: a powerful detector for future neutrino experiments and proton decay searches, Nucl. Phys. B Proc. Suppl. 154 (2006) 163;

A. Meregaglia and A. Rubbia, Neutrino oscillation physics at an upgraded CNGS with large next generation liquid argon TPC detectors, JHEP 11 (2006) 032 hep-ph/0609106;

A. Bueno et. al., Nucleon decay searches with large liquid argon TPC detectors at shallow depths: atmospheric neutrinos and cosmogenic backgrounds, JHEP 04 (2007) 041, hep-ph/0701101.

[10] T. Marrodán Undagoitia et al., Low energy neutrino astronomy with the large liquid scintillation detector LENA, Prog. Part. Nucl. Phys. 57 (2006) 283.

[11] A. de Bellefon et. al., MEMPHYS: a large scale water Cerenkov detector at Frejus, hep-ex/0607026. 
[12] C.K. Jung, Feasibility of a next generation underground water Cherenkov detector: UNO, AIP Conf. Proc. 533 (2000) 29 hep-ex/00050 46;

UNO collaboration, UNO White-paper: physics potential and feasibility of UNO, SBHEP-01-03 (2000) http://nngroup.physics.sunysb.edu/uno/.

[13] K. Nakamura, Hyper-Kamiokande: a next generation water Cherenkov detector, Int. J. Mod. Phys. A $\mathbf{1 8 ( 2 0 0 3 ) 4 0 5 3 .}$.

[14] P. Benetti et al., Detection of energy deposition down to the keV region using liquid xenon scintillation, Nucl. Instrum. Meth. A 327 (1993) 203.

[15] P. Benetti et al., First results from a dark matter search with liquid argon at $87 \mathrm{~K}$ in the Gran Sasso underground laboratory, Astropart. Phys. 28 (2008) 495.

[16] E. Aprile et. al., Simultaneous Measurement of Ionization and Scintillation from Nuclear Recoils in Liquid Xenon as Target for a Dark Matter Experiment, Phys. Rev. Lett. 97 (2006) 081302 astro-ph/0601552.

[17] L. Bruschi et al., Measurement of a Barrier for the Extraction of Excess Electrons for Liquid Helium, Phys. Rev. Lett. 17 (1966) 682; Transmission of negative ions through the liquid-vapour surface in neon, J. Phys. C 8 (1975) 1412;

A.F. Borghesani et al., Electron transmission through the Ar liquid-vapor interface, Phys. Lett. A 149 (1990) 481.

[18] CD-Adapco Group, User Guide, Star-CD V4.02 (2006).

[19] C. Carpanese et. al., ARIANNA: the Icarus experiment readout module, IEEE Trans. Nucl. Sci. 45 (1998) 1804; Daedalus: a hardware signal analyser for Icarus, Nucl. Instrum. Meth. A 409(1998) 294;

S. Centro et. al., Performance evaluation of a hit finding algorithm for the ICARUS detector, Nucl. Instrum. Meth. A 412 (1998) 440.

[20] V. Radeka et. al., Design of a charge sensitive preamplifier on high resistivity silicon, IEEE Trans. Nucl. Sci. 35 (1988) 155;

S. Centro, Signal amplification for high capacitance ionization chambers, Nucl. Instrum. Meth. A 315 (1992) 404;

C. Bacci et al., A hybrid charge sensitive amplifier for high capacitance detectors, Nucl. Instrum. Meth. A 273 (1988) 321.

[21] S. Centro et. al., Low-noise BiCMOS front-end and fast analogue multiplexer for ionization chamber, Nucl. Instrum. Meth. A 409(1998) 300. 